\title{
NISTIR 7096
}

\section{The Virtual Cement and Concrete Testing Laboratory Consortium}

Annual Report 2003

Editor: Jeffrey W. Bullard
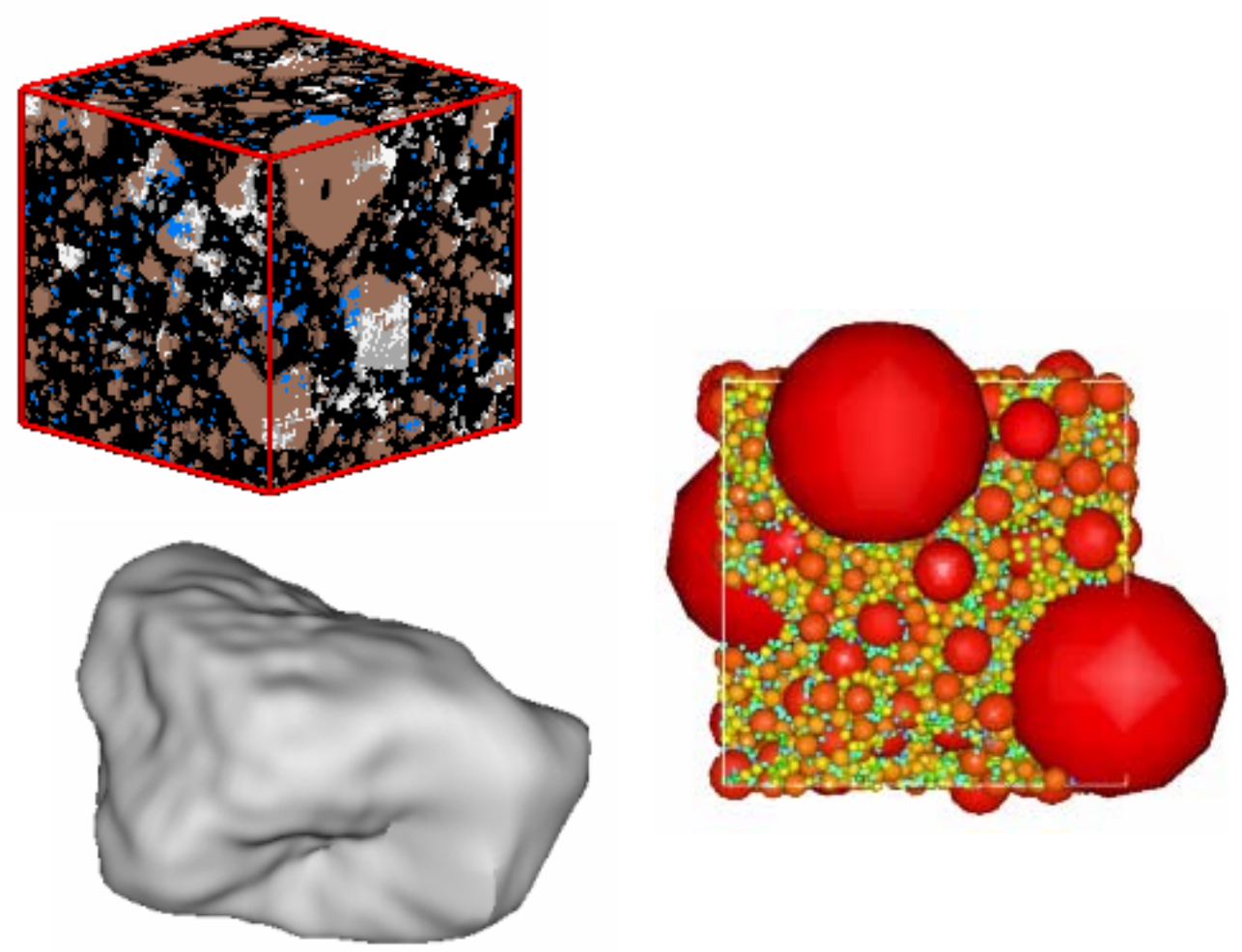

National Institute of Standards and Technology Technology Administration, U.S. Department of Commerce 
NISTIR 7096

\section{The Virtual Cement and Concrete Testing Laboratory Consortium}

\section{Annual Report 2003}

Editor: Jeffrey W. Bullard Building and Fire Research Laboratory

January 2003

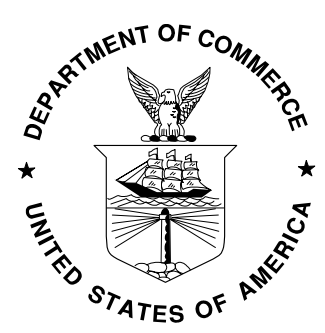

U.S. DEPARTMENT OF COMMERCE

Donald L. Evans, Secretary TECHNOLOGY ADMINISTRATION Phillip J. Bond, Under Secretary of Commerce for Technology 


\section{Contents}

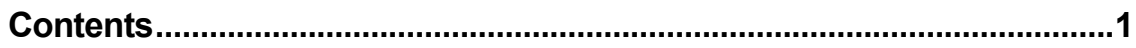

Virtual Cement and Concrete Testing Laboratory Consortium ................3

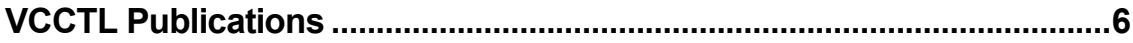

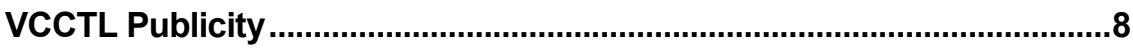

VCCTL Consortium Members .................................................................10

Materials Characterization ..........................................................................11

Hydration Research.........................................................................16

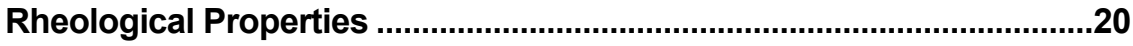

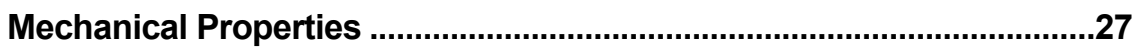

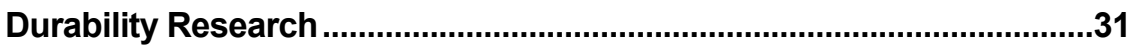

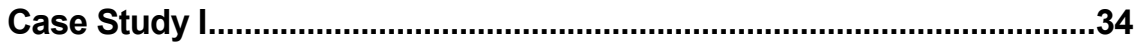

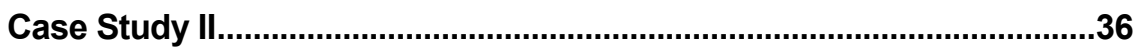

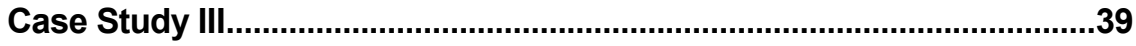

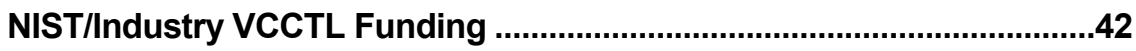

NIST Equipment and Facilities.............................................................42

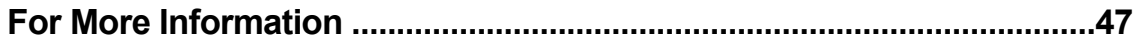




\section{DISCLAIMER}

Certain trade names or company products are mentioned in the text to specify adequately the experimental procedure and equipment used. In no case does such identification imply recommendation or endorsement by the National Institute of Standards and Technology, nor does it imply that the equipment is the best available for the purpose. 


\title{
Virtual Cement and Concrete Testing Laboratory Consortium
}

\author{
Annual Report 2003
}

\section{Background}

The Virtual Cement and Concrete Testing Laboratory (VCCTL) Consortium was formed in January 2001. Headquartered in the Building and Fire Research Laboratory (BFRL) at the National Institute of Standards and Technology (NIST), the consortium originally consisted of three NIST laboratories: BFRL, the Information Technology Laboratory (ITL), and the Materials Science and Engineering Laboratory (MSEL), and six industrial members: Cemex Trademarks Worldwide, Ltd., Dyckerhoff AG, Holcim (US) Inc., Master Builders Technologies Inc. (MBT), the Portland Cement Association (PCA), and W.R. Grace \& Co. The overall goals of the consortium were-and continue to be-to develop a virtual testing system that will reduce the amount of physical concrete testing needed and expedite the research and development process. This will result in substantial time and cost savings to the concrete construction industry as a whole.

The VCCTL Consortium Oversight Board, consisting of one representative from each member organization and the VCCTL Consortium Manager from NIST, governs the activities and direction of the consortium. The oversight board meets twice each year to review research progress and set the scope and agenda of future research. Within each industrial participant's laboratory, one researcher is assigned to participate in the research programs of the consortium. Once each year, the most recent version of the VCCTL system software is installed on a desktop computer at each participating member's laboratory.

The ultimate goal of the consortium is to address durability and service life prediction, but the members recognized that first efforts must be concentrated towards enhancing the current microstructure models and property calculations. Therefore, the three major initial research topics of the consortium were: 1) enhancements to the cement hydration and microstructure development model to consider additional supplementary materials such as slags and limestones and the prediction of pore solution concentrations, 2) computation of the elastic properties (elastic modulus, creep, shrinkage) of three-dimensional microstructures, and 3) experimental measurement and computer modeling of the rheological properties (viscosity, yield stress) of cement-based materials. 
This document reports the activities of the VCCTL Consortium in 2003.

\section{Overview of Activities in 2003}

The 2003 calendar year represents the third and final year (hereafter called Year 3) of the initial three-year consortium term. At the start of Year 3, the consortium was comprised of eight industry members: Cemex Trademarks Worldwide, Ltd., Dyckerhoff AG, Holcim (US) Inc., the International Center for Aggregates Research (ICAR), the Portland Cement Association (PCA), Sika Technology AG, Verein Deutscher Zementwerke eV(VDZ), and W.R. Grace \& Co.. During Year 3, three new members were welcomed into the consortium:

- Association Technique de l'Industrie des Liants Hydrauliques (ATILH), Paris, FRANCE

- National Ready Mixed Concrete Association (NRMCA), Silver Spring, Maryland, USA (as of January 2004)

- Master Builder Technologies (MBT), Cleveland, Ohio, USA (as of January 2004).

The VCCTL Consortium continues to represent every major constituent of concrete-cement, aggregate, and chemical admixtures, the major manufacturers of concrete, and major research and service organizations.

One of our charter industry partners, Dyckerhoff AG, made major changes in its research focus and personnel in 2003. One of these changes was to discontinue participation in the VCCTL Consortium. Dyckerhoff had been one of the most active research participants throughout the first three years of the consortium. Their VCCTL representative, Dr. Claus-Jochen Häcker, contributed several key research advances, including modifying the dissolution behavior of sulfates in CEMHYD3D and the experimental measurement of elastic properties of cement paste phases. Dyckerhoff's participation and perspectives will be missed by the consortium.

Year 3 witnessed a number of important research advances in each of the VCCTL's major focus areas. These advances are summarized in the following table.

\begin{tabular}{|l|l|}
\hline $\begin{array}{l}\text { Materials } \\
\text { Characterization }\end{array}$ & $\begin{array}{l}\text { Direct measurement of gypsum PSD in cement powder; } \\
\text { tomographic analysis of aggregates from } 19 \text { sources, } \\
\text { characterization of cement particle shapes }\end{array}$ \\
\hline Hydration & $\begin{array}{l}\text { Incorporation of real cement particle shapes, integration } \\
\text { of actual cement PSD into models; calculation of one- } \\
\text { pixel particle bias; improvements in fly ash reaction } \\
\text { kinetics }\end{array}$ \\
\hline Elasticity & $\begin{array}{l}\text { Validation of effective medium theory for mortar and } \\
\text { concrete elastic moduli predictions; exploration of } \\
\text { compressive strength predictions from elastic moduli }\end{array}$ \\
\hline
\end{tabular}




\begin{tabular}{|l|l|}
\hline Rheology & $\begin{array}{l}\text { A simulation of a parallel plate rheometer was } \\
\text { developed; and interparticle interaction potentials were } \\
\text { introduced in the DPD model }\end{array}$ \\
\hline $\begin{array}{l}\text { Three models were made available through the NIST } \\
\text { website; progress was made in development of an } \\
\text { accelerated test for determining the sulfate resistance of } \\
\text { a cement. }\end{array}$ \\
\hline
\end{tabular}

As in previous years [1,2], most of this progress was made possible only by collaborative efforts between NIST and the industry partners, a fact that demonstrates once again how critical the consortium arrangement has been to accelerating research.

Further enhancements also were made in Year 3 to the web-based VCCTL interface, augmenting the major changes made in Year 2. Among these are the ability to search online for desired files needed as input in the user forms, added flexibility in the specification of microstructure parameters in CEMHYD3D, and links to common next steps at the bottom of most forms to make navigation of the forms easier.

\section{References}

[1] "The Virtual Cement and Concrete Testing Laboratory Consortium Annual Report 2002," NISTIR 6962, U.S. Department of Commerce. Edited by J.W. Bullard, January 2003.

[2] "The Virtual Cement and Concrete Testing Laboratory Consortium Annual Report 2001," NISTIR 6840, U.S. Department of Commerce. Edited by D.P. Bentz, December 2001. 


\section{VCCTL Publications}

\section{Technical Notes}

- Technical Note VCCTL-01: Estimation of the Degree of Hydration of Portland Cement by Determination of the Non-Evaporable Water Content

- Technical Note VCCTL-02: SEM/X-ray Imaging of Cement Powders

- Technical Note VCCTL-03: Quantitative Determination of Calciumsulfate Dihydrate and Calciumsulfate Hemihydrate in Cement by Means of Thermogravimetric Analysis (from Dyckerhoff)

- Technical Note VCCTL-04: Estimation of the Degree of Hydration of Cement by Measurement of Chemical Shrinkage

- Technical Note VCCTL-05: Rheological measurement of cement paste/mortar using a parallel plate rheometer

- Technical Note VCCTL-06: Standard Method for Measuring the Particle Size Distribution of Hydraulic Cement and Related Compounds by Light Scattering

- Technical Note VCCTL-07: Wet/Dry Cycling Method for Determining Resistance to Sulfate Attack (due in 2004)

- Technical Note VCCTL-08: Pore solution extractor (due in 2004)

\section{Software Notes}

- Software Note VCCTL-01: CONCRETEVIEW software

- Software Note VCCTL-02: Software for Hydration/Drying of 2-mm Thick Microstructures

- Software Note VCCTL-03: Computation of Cement Paste Elastic Properties

- Software Note VCCTL-04: Software for the Degradation of Hydrated Microstructures Due to Magnesium Sulfate Attack 


\section{Manuals}

- D.P. Bentz and G.P. Forney, "User's Guide to the NIST Virtual Cement and Concrete Testing Laboratory. Version 1.0". NISTIR 6583. U.S. Department of Commerce, November 2000.

- J.W. Bullard, “User's Guide to the NIST Virtual Cement and Concrete Testing Laboratory. Version 3.0". Distributed to consortium members as part of the VCCTL Version 3.0 software distribution.

- J.W. Bullard, "User's Guide to the NIST Virtual Cement and Concrete Testing Laboratory. Version 1.1". NISTIR 6984. U.S. Department of Commerce, March 2003.

\section{Reports}

- "The Virtual Cement and Concrete Testing Laboratory Consortium Annual Report 2001". Edited by D.P. Bentz. NISTIR 6840. U.S. Department of Commerce, December 2001.

- "The Virtual Cement and Concrete Testing Laboratory Consortium Annual Report 2002". Edited by J.W. Bullard. NISTIR 6962. U.S. Department of Commerce, January 2003. 


\section{VCCTL Publicity}

NEW 2003 E.J. Garboczi, "The Virtual Cement and Concrete Testing LaboratoryProgress Report and ASTM Relevance." Presentation to ASTM Committees C01 and C09, Tampa, FL. (December 10, 2003).

NEW 2003 J.W. Bullard, et al., "Virtual Cement and Concrete". Chapter 10 in Innovations in Portland Cement Manufacture. Edited by J. Bhatty and S. Kosmatka. Portland Cement Association, in press.

NEW 2003 R.C. Meininger, “Virtual Aggregates,” Rock Products. October 1, 2003. Available online at http://rockproducts.com/ar/rock virtual aggregates/

NEW 2003 “NRMCA Participates in Development of Virtual Concrete Testing Lab." NRMCA E-NEWS. November 24, 2003.

NEW 2003 "Virtual Cement and Concrete-The Coming Revolution in Concrete Technology." Special symposium held at the ACI Spring 2003 Convention, Vancouver, Canada. April 1, 2003.

- Presentation to the ACI Strategic Development Council as part of the New Technology Showcase, Orlando, FL (November 18, 2002). "VCCTL: A WebBased Virtual Cement and Concrete Testing Laboratory."

- Special presentation by E.J. Garboczi to the Research Committee of the National Ready-Mixed Concrete Association, Arlington, VA (October 6, 2002). "VCCTL: A Web-Based Virtual Cement and Concrete Testing Laboratory

- Special 90-minute presentation by J.W. Bullard and E.J. Garboczi at the Portland Cement Association meeting, Chicago, IL (September 16, 2002). "VCCTL: A Web-Based Virtual Cement and Concrete Testing Laboratory."

- Daily Commercial News and Construction Record (September 13, 2002). "Facility Provides Virtual Testing for Concrete."

- Special symposium organized by G.J. Frohnsdorff and C. Ferraris, presented to ASTM Committees C-01 and C-09, Salt Lake City, UT (June 26, 2002). "The Virtual Cement and Concrete Testing Laboratory."

- Concrete Construction (November, 2001). "Virtual Concrete Laboratory" 
- The Concrete Producer (October, 2001). “E-Concrete: Let's Try a Little Left Thinking"

- Government Computer News (August 27, 2001). "NIST Team Sees in Stereo" (available at http://www.gcn.com/20 25/news/16941-1.html)

- Engineering News Record (June 25, 2001). "Website Aims to Cut Concrete Analysis" (available at http://www.enr.com/itnews/it62501e.asp )

- Civil Engineering (June, 2001). “Virtual Concrete Lab Saves Time, Reduces Material Cost"

- NIST Technology at a Glance (Spring, 2001). “Co-Op Corner, Virtual Cement Laboratory"

- $\quad$ NIST Update (February 20, 2001). “Virtual Lab Consortium to Test Concrete and Cement Formulas"

- Concrete Technology Today- PCA (December, 2000). “ The Future of Materials Testing? Virtual Testing of Concrete Will Save Time and Money"

VCCTL Newsletter. In Year 2, NIST began publishing a VCCTL Newsletter, which is distributed to members of the VCCTL Consortium and to users who login to the public domain site for the VCCTL software (Version 1.1). The newsletter highlights applications by users of the public domain version and also gives periodic tips for using the software. The VCCTL Newsletter is intended as a source of useful information as well as a publicity tool for the consortium. 


\section{VCCTLConsortium Members}

NIST

Dr. Jeffrey W. Bullard (BFRL)

Dr. Judith Devaney (ITL)

Dr. Chiara F. Ferraris (BFRL)

Dr. Edward J. Garboczi (BFRL)

Mr. John Hagedorn (ITL)

Mr. Peter Ketcham (ITL)

Dr. Nicos S. Martys (BFRL)

Mr. Steve Satterfield (ITL)

Mr. Paul Stutzman (BFRL)

Cemex Trademarks Worldwide, Ltd

Ms. Karen Ornelas

Mr. Javier Vazquez

Dr. Davide Zampini

Verein Deutscher Zementwerke eV (VDZ)

Dr. Martin Schneider

Dr. Georg Locher

Dr. Maria Teresa Alonso
Holcim (US) Inc.

Mr. Barry Descheneaux

Mr. Al Innis

Portland Cement Association

Dr. Paul Tennis

W.R. Grace \& Co.- Conn.

Dr. Vijay Gupta

Dr. David Myers

Dyckerhoff AG

Dr. Claus-Jochen Häcker
Association Technique de l'Industrie des Liants Hydrauliques (ATILH)

Dr. Alain Capmas

Prof. Denis Damidot (Mines de Douai)

\section{Sika Technology AG}

Dr. Robert Flatt
International Center for Aggregates Research (ICAR)

Prof. David W. Fowler (U. Texas at Austin) 


\section{Materials Characterization}

The properties of materials depend upon their internal structure. In turn, the properties influence the performance of a material, both during manufacture and in ultimate service [1].

\section{Background}

Without meticulous characterization of the starting materials, it is practically impossible to formulate fundamental microstructure models of the hydration and properties of a cement paste, mortar, or concrete. One of the signature features and strengths of the CEMHYD3D hydration model is its incorporation of microstructural information with unprecedented levels of detail.

Proper characterization of cement-based materials is not a trivial task. The starting powders typically consist of a wide distribution of particle sizes, each particle of which is composed of multiple phases with variable chemical compositions. Many products of hydration are amorphous or poorly crystalline, and these may be finely intermixed. An additional complication is that some microstructural characteristics have not been rigorously defined. In some cases it has not been possible even to properly calibrate measurements, like those of particle size distribution (PSD), because of the absence of an accepted reference material or standard methodology.

Recognizing the critical importance of materials characterization for predictions of microstructure development, physical properties, and durability, the consortium has continually sought to achieve greater accuracy and detail in these types of measurements, and to supply robust, calibrated measurement techniques where those have been lacking.

\section{Activities in Years 1 and 2}

Particle Size Distribution. In collaboration with ASTM Subcommittee C01.25, it was established that there was no standard test for measuring the PSDs of cements and no reference material existed. NIST was instrumental in 1) analyzing the data that were already available from a round-robin conducted by ASTM [2] and 2) organizing a second, more extensive, round-robin and analyzing the data [3]. The main result was to establish that: 1) the most used method by the cement industry is the laser diffraction technology; 2) the particle size distribution of the standard reference material (SRM), cement SRM 114p, was established and now is included in the certificate provided by NIST with the material; 3) the basis of a standard procedure was developed. Therefore, now the industry has a reference material that can be used to calibrate an instrument or to validate the laser diffraction procedure used in a laboratory. 
The efficacy of calcium sulfate in Portland cement, for controlling $C_{3} A$ hydration and microstructure development, has been demonstrated to depend on its spatial distribution and specific surface area [4]. Because both of these factors are expected to depend on the PSD of the calcium sulfate component, research was initiated in Year 2 to find and validate techniques for measuring the PSD of calcium sulfate after it has been blended/interground with clinker. By the end of Year 2, a novel approach using the wet laser diffraction technique had been identified. More details on the technique and its validation are given in the next section.

SEM/X-ray Imaging. NIST has pioneered the use of SEM/X-ray imaging for the quantitative characterization of cement powders [5]. This analysis produces a 2-D image of the cement powder in which each pixel is identified as one of the major phases of portland cement.

\section{Research in Year 3}

Particle Size Distribution. To pursue further the goal of providing industry with a method to measure the PSD of cement, two actions were pursued in year 3 . The first one is related to the SRM 114p cement, whose stocks are fast diminishing. Therefore, NIST initiated a procedure to produce the next generation SRM, SRM 114Q. This procedure includes the purchase of a large stock of cement, the homogeneisation of the material, the production of 100000 vials in sealed containers, and the characterization of the material by a round-robin. This process is expected to be completed in 2004. NIST developed a standard procedure that was provided to the members as Technical Note \#6. This standard was presented to ASTM subcommittee C01.25 and to the Task Group on PSD but was rejected because the Task Group wants to develop a standard that includes all possible methods, i.e., SEM, EZS, sieving, and sedimentation. The Task Group wants to use the SRM 114 curves developed for laser diffraction as a reference for all other methods. NIST's opinion is that this is not a valid approach because none of the methods measure the PSD directly. They all rely on assumptions (spherical particles, perfect dispersion, refractive indexes, etc.) and models to determine the PSD from the measured values. Also, from the round-robins conducted and a survey of Cement and Concrete Reference Laboratory (CCRL) members, it is clear that no statistical values could be obtained as the users of methods other than laser diffraction are few (1-3 at most). We will pursue the possibility of convincing ASTM to consider developing a test for PSD by laser diffraction.

\section{PSD of Gypsum in Cement}

The goal was to be able to determine the PSD of gypsum after it has been mixed or interground with clinker. This implies finding a method that can either physically separate the gypsum from the clinker or optically isolate the gypsum. Several unsuccessful tentative attempts were made to physically separate the gypsum using the density difference between the clinker particles and the gypsum. The method adopted was to use the laser diffraction device instead. The principle of the method is to render some particles, the gypsum for instance, "invisible" by matching the real refractive index with the medium. 


\begin{tabular}{|c|c|}
\hline Material & $\begin{array}{c}\text { Real Refractive } \\
\text { Index }\end{array}$ \\
\hline Isopropanol (IPA) & 1.39 \\
\hline Benzyl Alcohol (BA) & 1.54 \\
\hline Clinker & 1.7 \\
\hline Gypsum & 1.525 \\
\hline
\end{tabular}

Table1: Real components refractive indices for dispersants and cement components. Values are reasonably consistent over a range of wavelengths. For the solid components, the values reported are averages over all phases and crystallographic orientations

Preliminary results show that the method is valid. A gypsum and a ground clinker were blended and measured in IPA and in benzyl alcohol (BA). The various real refractive indices of interest are given in Table 1.

A mixture of gypsum and clinker dispersed and measured in BA will result in only the clinker PSD being measured, while in IPA both are measured. By properly weighting and subtracting the BA curve from the IPA curve, one can in principal obtain the gypsum PSD. The same technique could be used for other components if the relevant refractive indices were known.

Figure 1 shows an example obtained from an interblended clinker and gypsum. Inside the small circle in Figure 1, the calculated gypsum distribution is very

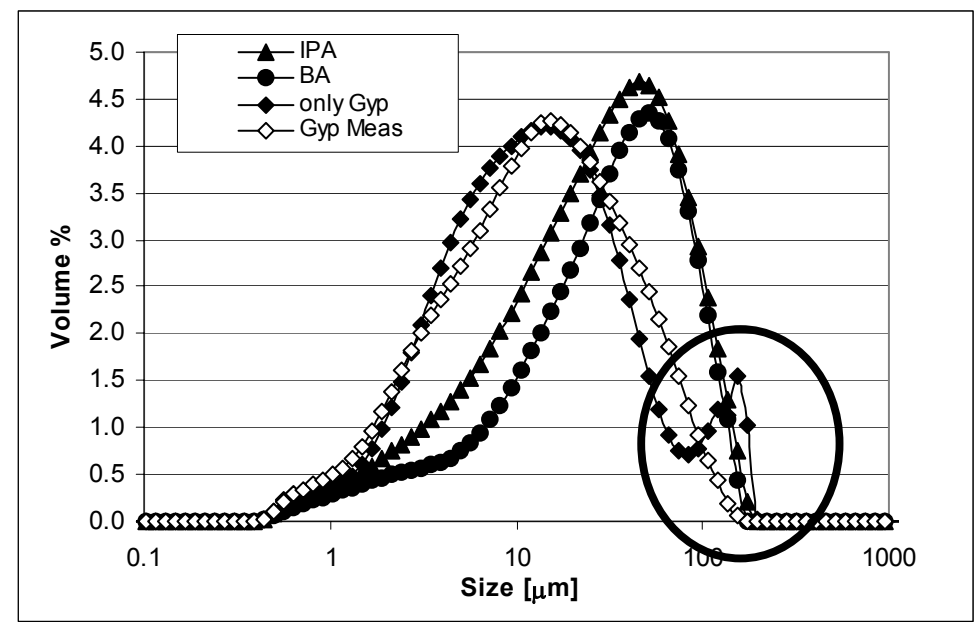

Figure 1: Gypsum PSD in interblended cement. In the circle are the data that are suspicious (see text for explanation).

different from the measurement of gypsum alone. This error is probably due to the small differences between IPA and BA data. The error is amplified by the multiplication to obtain the correct compound relative ratios. It also could be due to 
systematic errors in the measurements. NIST is investigating methods to eliminate these errors.

Aggregate Shape Characterization. In Year 2, we demonstrated how to characterize aggregate shape using a combination of $x$-ray tomography and spherical harmonics [6]. In Year 3, we further validated and streamlined this approach [7]. At the May 2003 VCCTL meeting, a call was put out for consortium companies to send several aggregates to NIST, which was going to pay The Pennsylvania State University Center for Quantitative Imaging to collect X-ray tomographs of these aggregates (about $\$ 25 \mathrm{~K}$ ). After the images were analyzed, the shape data would be added to the aggregate database in VCCTL 4.0. Several companies sent aggregates (Cemex, Holcim, PCA, ATILH, Sika, ICAR). There was a several month delay due to equipment problems at Penn State, which were resolved. As of this report, almost all the aggregates have been scanned and processed. All this shape information will be put into the VCCTL 4.0 database, along with size, elastic moduli, absorption, and maximum packing information (to the extent obtainable).

Figure 2 shows the kind of statistical analysis that can be done after the shape information is obtained. The graph was done for an ICAR crushed granite coarse aggregate. The solid line is an exact theoretical curve for a sphere, surface area $\mathrm{S}=4.84 \mathrm{~V}^{2 / 3}$, where $\mathrm{V}$ is the volume of the particle. The dashed line is a curve of the same form fit to data for a GR sand, $S=8.1 \mathrm{~V}^{0.63}$. The similarity of the exponents is because the GR aggregates are ordinary 3-D particles. The larger coefficent in the power law is a measure of the non-sphericity of the particles.

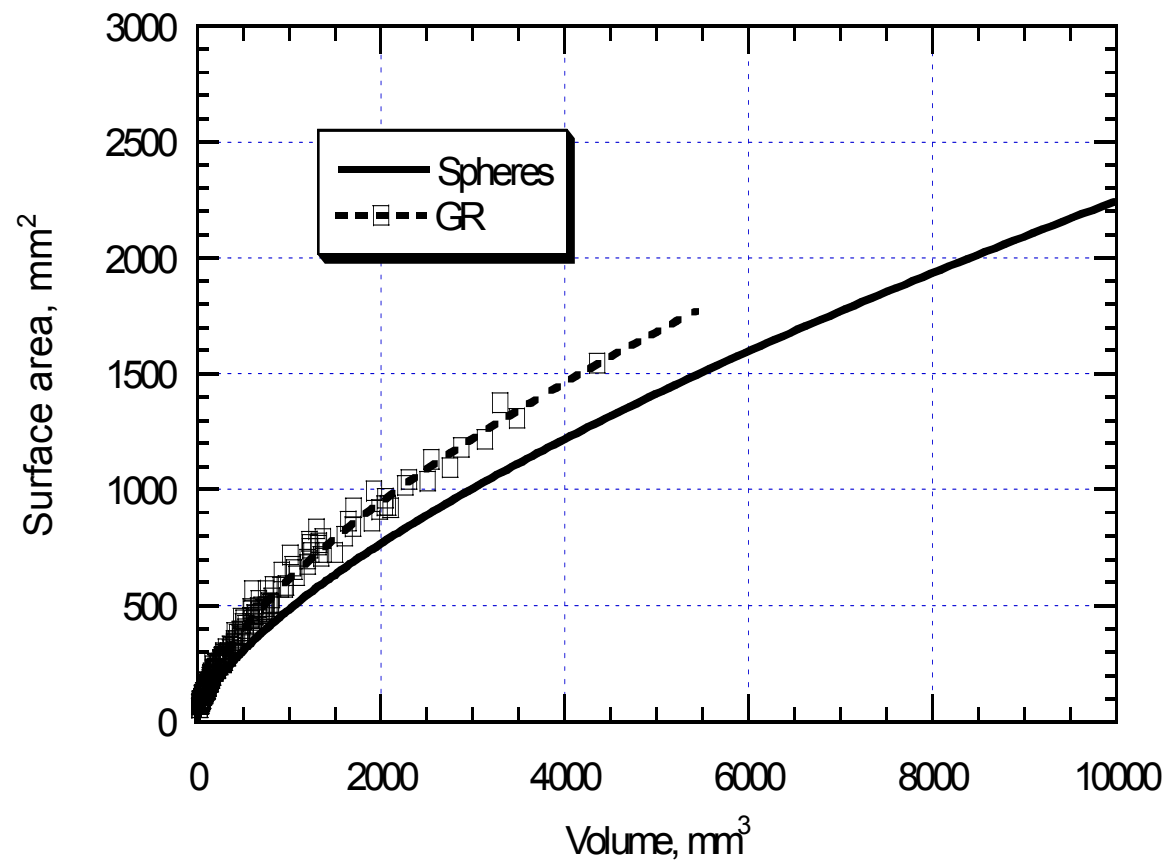

Figure 2: Surface area of particles as a function of their volume

The ultimate objective of this project is to provide a database of aggregate shapes for accurately modeling the macrostructure of concrete. Ongoing work is focusing on 
analyzing different types of aggregate (e.g. riverbed vs. crushed) for common characteristics and on enhancing the database.

\section{Future}

Particle Size Distribution. The SRM 114Q will be developed and further action will be taken to convince ASTM that a standard test is paramount for the industry. The optical method for isolating the PSD of gypsum in cement will be validated further and refined in the coming year.

Aggregates. The aggregates database will continue to expand and include more information (mineralogical composition, water absorption, etc.) for each entry. The VCCTL software, especially that dealing with rheological and mechanical properties of concrete, will access the database directly to read particle size and shape distributions that are required for predicting properties.

\section{References}

1. L.H. Van Vlack. p. 1 in Elements of Materials Science and Engineering. Fifth Edition. Addison-Wesley, Reading, MA, 1985.

2. C.F. Ferraris, V.A. Hackley, A.I. Aviles, and C.E. Buchanan, "Analysis of the ASTM Round-Robin Test on Particle Size Distribution of Portland Cement: Phase I". NISTIR 6883. U.S. Department of Commerce, May 2002. Available at http://ciks.cbt.nist.gov/ monograph/nist6883/nistir6883.htm .

3. C.F. Ferraris, V.A. Hackley, A.I. Aviles, and C.E. Buchanan, "Analysis of the ASTM Round-Robin Test on Particle Size Distribution of Portland Cement: Phase II". NISTIR 6931. U.S. Department of Commerce, December 2002.

4. F.J. Tang and E.M. Gartner, "Influence of Sulphate Source on Portland Cement Hydration," Adv. Cem. Res.1 [2] 67-74 (1988).

5. D.P. Bentz, P.E. Stutzman, C.J. Haecker, and S. Remond, "SEM/X-ray Imaging of Cement-Based Materials," 7th Euroseminar on Microscopy of Building Materials, Delft, The Netherlands, 457-466 (1999). Available online at http://ciks.cbt.nist.gov/monograph/eurosem/semcolor.html

6. E.J. Garboczi, "Three-Dimensional Mathematical Analysis of Particle Shape Using X-Ray Tomography and Spherical Harmonics: Application to Aggregates Used in Concrete," Cem. Conc. Res. 32 [10], 1621-38 (2002). Available at http://ciks.cbt.nist.gov/monograph/paper134/mono134.html .

7. S. Erdogan, P.Quiroga, D. Fowler, H. Saleh, R.Livingston, E.J. Garboczi, P. Ketcham, and S. Satterfield, "Three-dimensional shape analysis of coarse aggregates: Methodology and preliminary results on several different coarse aggregates," in preparation (2004). 


\section{Hydration Research}

Mathematical modelling of hydration has the objective of quantifying knowledge of the hydration process and microstructure of the resulting material ...thus allowing the effects of changes in inputs or assumptions contained in the model to be examined [1].

\section{Background}

Because the physical, chemical, and durability properties of concrete are ultimately dependent on the composition and microstructure of its binder, a microstructurebased model of cement paste hydration is at the core of the Virtual Cement and Concrete Testing Laboratory software [2]. For several years, the 3-D model of hydration (CEMHYD3D) [3,4] has been recognized as the most extensive, complete, and robust cement paste microstructure model in the world. As hydration proceeds, a series of output files is created to track specific properties-chemical shrinkage, heat release, setting, capillary porosity percolation, $\mathrm{pH}$, and strength.

\section{Activities in Year 1}

During 2001, research focused on five subtopics: 1) modeling and experimental measurements on systems containing limestone fillers, 2) development of a framework for incorporating reactions for slag into CEMHYD3D, 3) modeling of the $\mathrm{pH}$ and ion concentration of the pore solution during hydration, 4) creation of options in CEMHYD3D for curing under sealed conditions and for specifying the nature of heat transfer between system and surroundings, and 5) addition of a supplementary model to monitor hydration/drying in $2 \mathrm{~mm}$-thick slabs [5].

\section{Activities in Year 2}

Research in 2002 concentrated primarily on incorporating in CEMHYD3D the influence of alkali species on hydration kinetics. The $\mathrm{pH}$ was used as the sole link between pore solution and hydration kinetics, and the dissolution rates of all clinker phases were assumed to change by a prescribed amount that depended only on $\mathrm{pH}$ and not on the clinker phase itself. This modeling work was performed in parallel with considerable experimental efforts at Cemex, Dyckerhoff, and NIST to measure the hydration rate of cements intentionally doped with alkali salts. Model predictions compared favorably with experiments on one cement, but were unsatisfactory for two other cements. Other modeling research included (1) extending CEMHYD3D to arbitrary system sizes and (2) automatic tracking of nonevaporable water content. 


\section{Research in Year 3}

Alkali effects. CEMHYD3D was modified to allow $\mathrm{pH}$ to influence the reaction rate of any given phase independently. Further experimental data on the influence of alkali species were obtained at W.R. Grace. Much of the apparent disagreement between model and experiment was discovered to be likely due to inadequate characterization of the dissolution rate of submicron cement particles. For cements containing fly ash, effort was placed on more accurately capturing the reaction rate of fly ash phases. This was accomplished by introducing a $\mathrm{pH}$-dependence on pozzolanic reaction rates to simulate the observed induction period of many fly ashes. Fly ash consumption predicted by the model now compares favorably with the limited experimental data that are available [6].

Dissolution of submicron particles. Since Year 1, a dissolution bias could be implemented to simulate accelerated dissolution of one-pixel particles. In Year 3, an equation was derived to explicitly calculate the required one-pixel dissolution bias based on the particle size distribution in the submicron range. This equation was incorporated into CEMHYD3D. This improvement goes a long way toward reconciling prior disagreements between predicted and experimental hydration rates, especially at early ages.

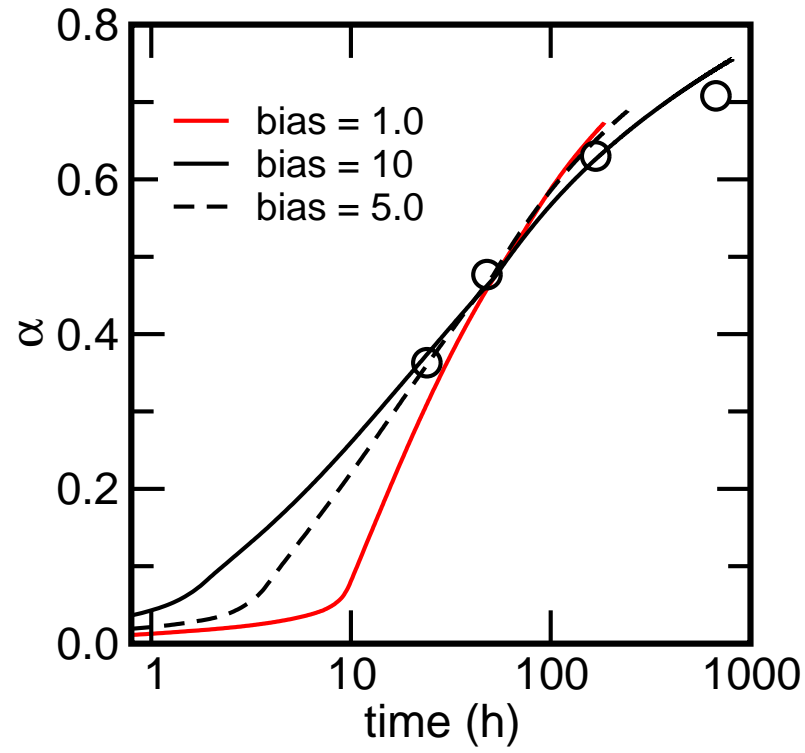

Figure 3: Influence of one-pixel dissolution bias onhydration kinetics of a Dyckerhoff Rumelange cement paste.

\section{Surface deactivation.}

Beginning in Year 2, users could choose to render inert for a specified time a certain fraction of all the surface sites in a cement, as a way to simulate the action of retarders. In Year 3, this feature was extended to allow deactivation of any fraction of any surface type, including hydration products such as portlandite.

Real particle shapes. The Visible Cement Data Set [7] was used to extract and reconstruct real shapes of cement particles (clinker and gypsum). The source of the cement particle images was CCRL cement 133. Cylinderical samples $1 \mathrm{~mm}$ in diameter were made by Dale Bentz and imaged at the ESRF facility, Grenoble, France. The imaging was done at a resolution of 0.95 $\mu \mathrm{m} /$ voxel length, using $1024^{3}$ voxels. More details and actual data can be found at http://visiblecement.nist.gov. Many images were taken at various degrees of hydration. Only the earliest age, $3 \mathrm{~h}$, was used for this work. At this point, set had just occurred, so that stable imaging could be done, but not enough hydration had taken place to significantly disturb the shape of the particles of interest, of diameter $5 \mu \mathrm{m}$ and above. Real shapes can now be used to construct 3-D microstructures in 
exactly the same way as spherical particles have been used in the past (see Case Study I)

New phases. Lime $(\mathrm{CaO})$ was added to CEMHYD3D as a possible cement phase. Using available thermodynamic data as a guide, $\mathrm{CaO}$ was assumed to dissolve readily in water to form solid $\mathrm{CH}$. Solubility data for $\mathrm{CaO}$ in water was compared to that for gypsum and portlandite to assign a value for the relative dissolution probability of free lime. However, this value should be examined closely and adjusted as more experimental data become available. The orthorhombic polymorph of $\mathrm{C}_{3} \mathrm{~A}$, which is known to be more reactive in water than the cubic form, also was added as a new phase. The orthorhombic form of $\mathrm{C}_{3} \mathrm{~A}$ currently is set to dissolve $1.5 \times$ more readily than the cubic form.

Centralization of model parameters. As a way to manage the complexity of CEMHYD3D more effectively, the adjustable parameters used within the model were collected together in a single input file. Also, each parameter was documented in a separate file. These parameters, and their documentation, now may be viewed and edited through the user interface and then submitted for any given hydration simulation, without the need to recompile the code.

\section{Future}

CEMHYD3D has been shown to be an extremely powerful and useful tool for modeling the microstructure development of cement pastes. Nevertheless, the model in its current form is limited in its ability to accurately capture the kinetics of hydration. The reason for this is two-fold: (1) certain important mechanisms that control hydration rates, especially at late ages, are not included in the model, and (2) the model has no intrinsic time scale with which to dictate the progression of time. The model compensates by using a user-specified time conversion factor and an assumed parabolic relation between model cycles and time. A major goal in Phase II of the consortium will be to endow the hydration model with more realistic kinetics. A second major objective of hydration research is to more accurately link pore solution chemistry to cement hydration and to make the model extensible to new cement phases or mineral admixtures.

Reaching these goals in Phase II will require a significant overhaul of the structure and algorithms of CEMHYD3D. The program likely must be rewritten in an objectoriented language, which in turn will involve a restructuring of data. Although this is a major undertaking with its own risks, it offers several outstanding opportunities. Chief among these is that, by making the next-generation model more extensible, the code should be easier to understand and modify in the future. Furthermore, details of validation will be embedded in the documentation to provide a concise, but complete, history of code development. Effort will be placed in the near term on correctly modeling ordinary portland cement before moving on to incorporate mineral admixtures.

\section{Major Goals for Phase II}

1. Mechanistic basis for time stepping to replace empirical time conversion scheme. 
2. Limited diffusion of selected ions through C-S-H and pozzolanic C-S-H gels

3. "On-the-fly" specification of new cement compounds or mineral admixtures.

4. More accurate, thermodynamically based tracking of pore solution composition, with corresponding links to reaction kinetics.

\section{References}

1. H.F.W. Taylor, p. 237 in Cement Chemistry. 2nd Edition. Thomas Telford Publishing, London, 1997.

2. D.P. Bentz and G.F. Forney, "User's Guide to the NIST Virtual Cement and Concrete Testing Laboratory. Version 1.0," NISTIR 6583, U.S. Department of Commerce, November 2000. Available at http://vcctl.cbt.nist.gov/vcctlman .

3. D.P. Bentz, "Three-Dimensional Computer Simulation of Cement Hydration and Microstructure Development," J. Amer. Ceram. Soc., 80 (1), 3-21, (1997). Available at http://ciks.cbt.nist.gov/monograph/AmCeram/ACSmain.htm .

4. D.P. Bentz, "CEMHYD3D: A Three-Dimensional Cement Hydration and Microstructure Development Modelling Package. Version 2.0," NISTIR 6485, U.S. Department of Commerce, April 2000. Available at http://ciks.cbt.nist.gov/ bentz/cemhyd3dv20 .

5. D.P. Bentz, “The Virtual Cement and Concrete Testing Laboratory Consortium Annual Report 2001," NISTIR 6840, U.S. Department of Commerce, December 2001.

6. X. Feng, et al., "Estimation of the Degree of Hydration of Blended Cement Pastes by a Scanning Electron Microscope Point-Counting Procedure Cem. Conc. Res., in press.

7. D.P. Bentz, et al., “The Visible Cement Data Set,” J. Res. Natl. Inst. Stand. Technol. 107, 137-148 (2002). 


\section{Rheological Properties}

...fresh concrete is no longer considered as a material with a single parameter, called consistency or workability [1].

\section{Background}

At NIST, there is an ongoing program with the goal of predicting the rheological performance of concrete from its composition. A hierarchical approach, starting with cement paste and progressing to mortar and concrete, is used by which the rheological properties at one level are used as input to compute those at the next. This approach consists of the following steps:

1. measuring the rheological properties of the cement paste to determine the influence of chemical admixtures and supplementary cementitious materials,

2. measuring mortar rheological properties to determine the influence of air and sand content,

3. predicting rheological properties of concrete via computer simulation [2-4] using measurements on mortar and/or cement paste and information about the size and shape distributions of the coarse aggregates

A novel computer simulation approach allows NIST to take the rheological properties measured on mortar, add the coarse aggregates with their shape and PSD, and predict the concrete rheological properties. The advantage of this approach is that several tests could be done by one operator on cement paste or mortar in a day, while concrete testing requires several operators and much more material. The model is based on the theory of dissipative particle dynamics (DPD) [5-7], which combines aspects of cellular automata and molecular dynamics. The method described in Refs. [6,7] was modified by Martys at NIST for application to the flow of concrete. The model itself requires powerful computers, so NIST has built a database, included in Version 2.0 and expanded in Version 4.0 of the VCCTL software, that catalogues the relative viscosity, i.e. the ratio of the concrete viscosity to that of the mortar, of a concrete with a coarse aggregate defined by its size distribution.

The goals of the VCCTL Consortium in this area are 1) to establish standard tests for the influence of air and mix proportioning on the viscosity of mortar, 2) to validate the modeling approach for a wide range of systems with different supplementary cementitious materials (SCM) and chemical admixtures, and 3) to extend the VCCTL database to include realistic shapes and wider size distributions of common aggregates. 


\section{Activities in Year 1}

A methodology to test mortar was developed by modification of the parallel plate rheometer used previously for cement paste. A database was created to collect aggregate size distributions and graphs of relative viscosity versus aggregate concentration. A partial validation of the modeling approach was made possible by rheological measurements on mortar and concrete, provided by W.R. Grace using a BML rheometer [8,9] and Degussa/MBT using an IBB rheometer.

The DPD model was modified to include air as a second fluid phase. Air was introduced as spherical bubbles, which may subsequently deform under shear forces.

\section{Activities in Year 2}

From a combination of experimental measurements and simulation, it was established that the use of the relative viscosity concept allows the direct comparison of results from rheometers with different geometries. In other words, if the relative viscosity is considered instead of the plastic viscosity, data obtained with mortar using a small modified parallel plate rheometer or with concrete in two rheometers such as IBB and BML can be plotted on the same graph and seem to follow the same trend. This process is detailed in Ref. [10].

It was also shown that increases in the air content leads to a decrease in the relative viscosity both in mortar and concrete. This will allow determining the influence on concrete rheology of the air content introduced by a selected air-entraining agent (AEA) using mortar instead of concrete to perform the test. A first attempt to simulate the influence of air was also initiated.

The simulation was modified to allow for variation of the size distribution and shape of the coarse aggregate to determine its influence on the concrete flow. In addition, crushed aggregates shapes, based on X-ray tomography images, were introduced in the simulation. In agreement with field experience, the viscosity of a concrete with crushed aggregate was predicted by the simulation to be higher than a concrete with the same volume fraction of round coarse aggregate.

\section{Research in Year 3}

\section{Experimental}

Standard Measurement Method. One of the goals of this project is the prediction of the concrete rheology from the measurements on cement paste or mortar. The influence of air content is also one of the concerns. This year, a VCCTL Technical Note, which describes the methodology developed to measure the rheological properties of cement paste and/or mortar, was prepared. This is the starting point to allow other laboratories to measure the plastic viscosity of cement paste or mortar. The goal is eventually to standardize this methodology in ASTM. Extensive tests linking experimental results and modeling were conducted to better understand the critical geometrical parameters of the parallel plate rheometer used at NIST. This rheometer was modified to allow mortar measurements. The modification involves changing the top and bottom plates and the introduction of 


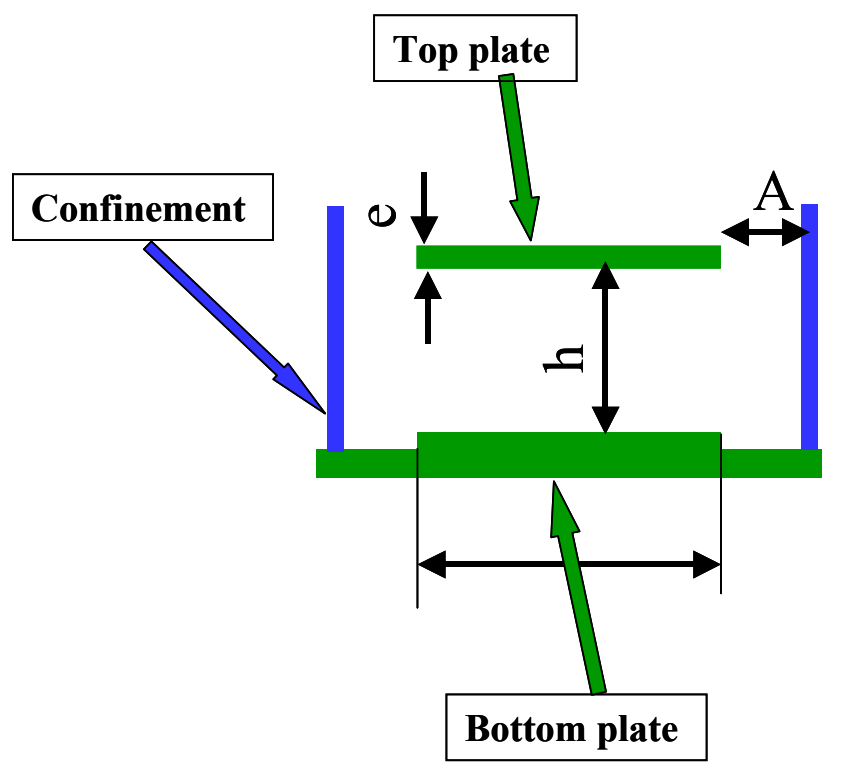

Figure 4: Schematic of the mortar rheometer. The parameters, $A, h$ and e are used to characterize the geometry

confinement walls (Figure 4). The use of a lattice Boltzman model of fluid flow determined that the three important parameters are $\mathrm{A}$ (distance between the wall and the top plate), $\mathrm{h}$ (gap between the two plates) and e (thickness of the top plate). Figure 4 shows that if the distance A is not correctly selected, the value of the viscosity might not be properly measured. In Figure 5, the data marked SIKA PP60M8 are two sets of data using A $=8 \mathrm{~mm}$ while the data marked SIKA PP60-M4 is for $\mathrm{A}=4 \mathrm{~mm}$. It is clear that due to the type of aggregates used the value of $8 \mathrm{~mm}$ for $\mathrm{A}$ yields data that are comparable to the concrete data while the smaller A results in a viscosity that is too high. A systematic study using standard oils was conducted to

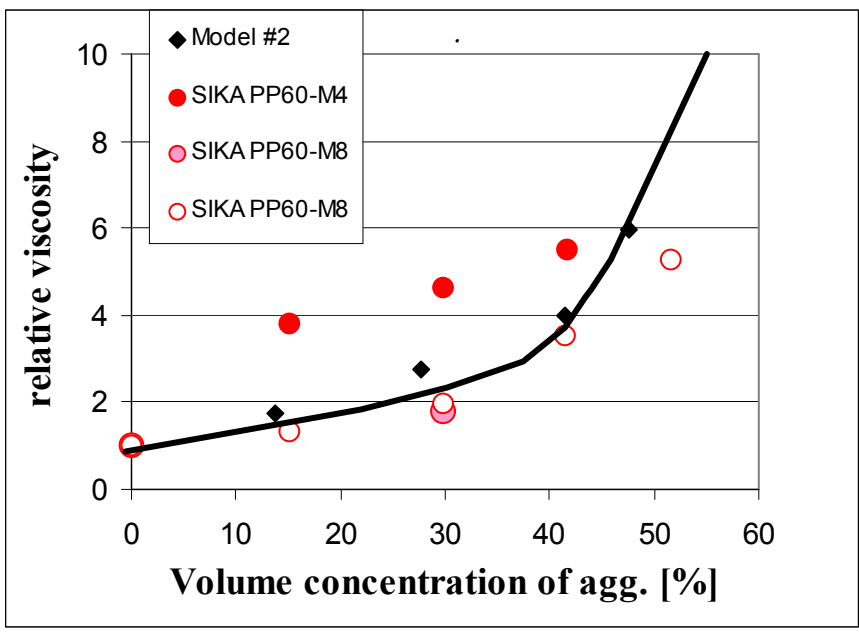

Figure 5: Influence of mortar rheometer geometry. 
validate the importance of the various parameters. This study is still in progress to measure oils with particles to further calibrate the modified rheometer. This work is being done in collaboration with Dr. Mette Geiker (DTU).

Air Content Influence: The second area of interest was the influence of air content on the rheology of mortar and concrete. W.R. Grace provided concrete data and NIST produced the mortar data. Figure 6 shows that the mortar measurements follow the same trend as the concrete data. It should be noted that, to compare concrete and mortar on the same graph in a consistent fashion, all plastic viscosity data are reported as relative viscosity. In this case, the relative viscosity is defined as the ratio of the viscosity measured in concrete/mortar with air to the same concrete/mortar with no air (or better with no AEA implying minimum air). Therefore, it can be shown, as previously found, that measurements on mortar mimic the concrete behavior.

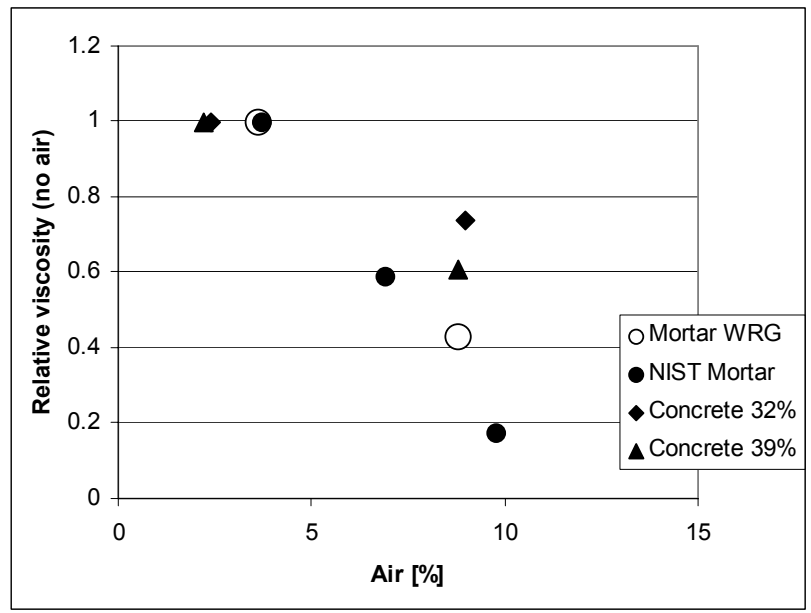

Figure 6: Comparison of measurements of concrete and mortar. The curve is not a modelbut is provided instead to guide the reader.

The next step is to model this behavior so as to predict the influence of air content on concrete flow. The first thing that should be noted is that one of the reasons for the viscosity to decrease with the increase in air content is that the particle concentration actually decreases with increasing air content. This can be visualized easily by the following equations:

- $\quad$ sand+coarse aggregate conc. $=($ sand + coarse aggregates vol. $) /$ Total Vol.

- Total Vol. $=\Sigma($ vol. of air, sand+coarse aggregates, cement, water)

Therefore, as the total volume is increased by the volume of the air, the sand+coarse aggregate concentration is reduced. If the new volume concentration is input and the semi-empirical Douglas equation for predicting relative viscosity is used, a reduction in the viscosity is observed as shown in Figure 7 . This first approximation 


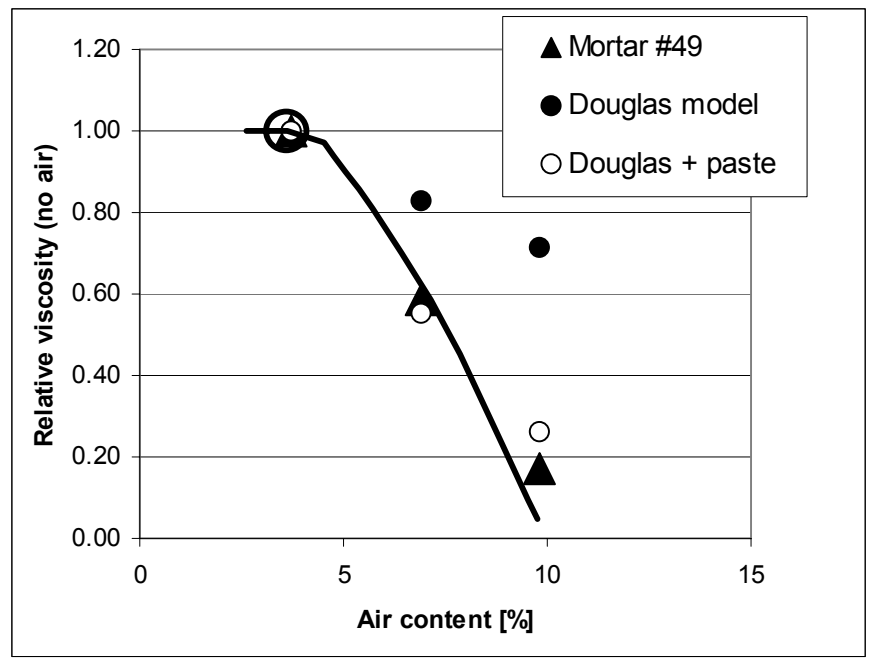

Figure 7: Comparison of mortar with Douglas equation

does not account for the total decrease seen in the experimental data. A further correction of the Douglas equation should be introduced to account for the changes in the paste due to air content (equivalent to an increase in $\mathrm{w} / \mathrm{c}$ ). Further tests are planned to systematically prove this methodology.

\section{Modeling}

In Year 3, significant progress was made in our modeling efforts. The rheology code was validated for many flow situations and it was also extended to account for interparticle interactions and flow in other geometries not unlike that used in some concrete rheometers.

Inclusion of Lubrication Forces The rheology code was extended to include lubrication forces for spherical particles. Lubrication forces can help produce a shear thickening effect at high solid fractions and shear rates so it was important to include this effect. Further, for the purposes of validating the rheology code, it was important to include this effect, because there are considerable experimental data using charge-neutralized colloidal systems with which to compare.

Reasonable agreement was found between well characterized experimental data and our simulation results. Also, reasonable agreement was found with other, well established, but more numerically intensive simulation approaches.

Examination of the Role of Particle Size Distribution In collaboration with Robert Flatt (Sika), we studied the role of particle size distribution on the rheological properties of sphere suspensions (see Case Study III). Several empirical theories for fitting data were investigated. We found that the Douglas equation provided very good fits for a fairly wide range of aggregate size distributions not unlike that used in concrete. 
Introduction of Interparticle Interactions Also in collaboration with Robert Flatt, an interaction potential was included in the simulations to account for agglomeration of particles. These preliminary investigations were encouraging, in that we were able to obtain a Bingham-like behavior in our colloidal system. As work on this project continues, we plan to introduce more refined models of interparticle interactions to account for the effect of steric hindrance from the use of high-range water-reducing admixtures (HRWRA).

\section{Future}

The simulation and measurement of rheological parameters of concrete need more study in two major areas:

1. Predict the influence of concrete composition (air content, aggregates shape, aggregate gradation and content, cement paste rheological) on the plastic viscosity by using a combination of measurements and simulation.

2. Predict the influence of concrete composition (air content, aggregates shape, aggregate gradation and content, cement paste rheological) on the concrete yield stress.

Ultimately, these two objectives will help select a mix design for its flow properties desired for a specific application while minimizing the trial mixes needed. This will be achieved by establishing a methodology to relate flow of a non-newtonian fluid in pre-selected geometries with its composition.

\section{Major goals for Phase II}

1. Validate the concept of multi-scale by determining whether the prediction of flow of concrete can be inferred from the mortar measurements and the mortar flow from the cement paste measurement.

2. Determine the influence of air on the rheological properties of concrete. Using a combination of theoretical and experimental means, establish a methodology to determine the influence of AEA on the rheological response of mortar and concrete.

3. Use simulations of concrete flow to determine the correlation between measurements obtained with concrete rheometers and the "real" plastic viscosity.

4. Development of a methodology to measure the yield stress in cement paste or mortar. This methodology will be a combination of measurements and simulation. The influence of the chemical admixtures, air content and SCM will be considered.

\section{References}

1. F. de Larrard, p. 81 in Concrete Mixture Proportioning: A Scientific Approach. Modern Concrete Technolgoy Series, Volume 9. Edited by A. Bentur and S. Mindess. E \& FN Spon, London (1999).

2. C.F. Ferraris, "Measurement of the Rheological Properties of Cement Paste: A New Approach," pp. 333-42 in Intl. RILEM Conference The Role of Admixtures in 
High Performance Concrete. Edited by J.G. Cabrera and R. Rivera-Villareal, (1999). Available at

http://ciks.cbt.nist.gov/monograph/rilem1999/rilemmain.htm .

3. C.F. Ferraris and N. Martys, "De la pâte de ciment au béton: modélisation et mesures expérimentales des propriétés rhéologiques," pp. 226-30 in Proc. Rhéologie Génie Civil et Environment, 36 ème Colloque du Groupe Francais de Rhéologie. Marne-la-Vallée, France, October 10-12, 2001.

4. C.F. Ferraris, F. de Larrard, and N. Martys, "Fresh Concrete Rheology", in Materials Science of Concrete VI. Edited by J.P. Skalny. American Ceramic Society, Westerville, $\mathrm{OH},(2001)$. Available at http://ciks.cbt.nist.gov/monograph/materialscience2000/msmain.htm .

5. R.D. Groot and P.B. Warren, "Dissipative Particle Dynamics: Bridging the Gap between Atomistic and Mesoscopic Simulation," J. Chem. Phys. 107, 4423-35 (1997).

6. P.J. Hoogerbrugge and J.M.V.A. Koelman, "Simulating Microscopic Hydrodynamic Phenomena with Dissipative Particle Dynamics," Europhys. Lett. 19, 155-60 (1992).

7. J.M.V.A. Koelman and P.J. Hoogerbrugge, "Dynamic Simulations of HardSphere Suspensions under Steady Shear," Europhys. Lett. 21, 363-68 (1993).

8. “Comparison of Concrete Rheometers: International Tests at LCPC (Nantes, France) in October 2000," NISTIR 6819, U.S. Department of Commerce. Edited by C.F. Ferraris and L. Brower, September 2001.

9. C.F. Ferraris, "Measurement of the Rheological Properties of High Performance Concrete: State of the Art Report," J. NIST Research 104 [5], 461-78 (1999).

10. C. F. Ferraris, N. S. Martys, "Relating Fresh Concrete Viscosity Measurements from Different Rheometers", J. Res. Natl. Inst. Stand. Technol. 108 (2003) 


\section{Mechanical Properties}

Cement paste is extraordinarily complex elastically, with many... different chemically and elastically distinct phases and a complex microstructure. This complexity further increases in concrete, as aggregates are added [1].

\section{Background}

The elastic behavior of engineering materials, especially those materials intended for structural applications, is a critical design factor for any project in which they will be used. Concrete is a random composite material over many length scales, consisting of phases, e.g. aggregate, C-S-H, and capillary porosity, which differ widely in their intrinsic elastic moduli. As a result, the effective elastic moduli of concrete cannot be predicted accurately by most of the analytic theories that are useful for other classes of materials.

Despite the little progress that has been made in predicting the elastic properties of cement and concrete, these properties are of great importance to industry. In addition to the significance of elastic response in the design of structures, there is growing interest in using the elastic moduli of a mortar or concrete as an estimator of other important mechanical properties such as its compressive strength.

(Prediction of strength from theoretical models is even more difficult than the prediction of elastic moduli). As a result, a major component of research within the VCCTL Consortium is directed toward developing robust numerical procedures that can predict the effective elastic moduli of cement, mortar, and concrete.

\section{Activities in Year 1}

A finite element package for computing the elastic moduli of composite materials that was written at NIST [2] and validated on a diverse range of material classes [36] was incorporated into Version 2.0 of the VCCTL software package. The finite element program operates directly on the same digital images that are generated by the other components of the VCCTL software, such as CEMHYD3D. This enables the true spirit of a "virtual lab" to be realized, because the user can create, hydrate and compute elastic properties of a microstructure, all within the same software package

As part of the validation effort, Dyckerhoff provided experimental measurements of the elastic moduli of a series of cement pastes, with w/c $=0.25$ to 0.60 , and cured under saturated conditions for various times. Dyckerhoff also provided experimental data on the elastic moduli of polycrystalline ettringite. NIST researchers gathered published values of the intrinsic elastic moduli of minerals commonly found in cement/cement paste. Agreement between model and 
experiment was quite reasonable (usually within $5 \%$ ) for later-age specimens, but more serious discrepancies were evident for early-age specimens.

\section{Activities in Year 2}

In Year 2, research focused on refining the predictions of the elastic moduli of cement paste at early ages (hours to several days after set), which resulted in much better agreement with experimental data in that time regime. Experimental data provided by Dyckerhoff were compared to the model predictions, and the error was shown to improve from nearly $200 \%$, for the original model, to no greater than $5 \%$ error for the corrected model at low w/c ratios. For higher w/c ratios $(\approx 0.6)$ the agreement is poorer, and current research effort is being placed on determining the source of the error and ways to improve those predictions.

\section{Research in Year 3}

The research in mechanical properties this year focused on (1) validating the effective medium theory for mortar and concrete elastic moduli predictions, and (2) exploring ways to predict the compressive strength from elastic moduli.

An effective medium theory was developed several years ago [7] to be applicable to mortar and concrete by directly taking into account the interfacial transition zone (ITZ). Essentially, the interfacial transition zone is averaged into each aggregate particle, making each particle slightly larger and with elastic properties averaged over a combination of aggregate and ITZ properties. The ITZ is treated as a uniform thickness, uniform property layer around each aggregate particle, which is of course an approximation. The sieve analysis of the aggregate is directly taken into account. An additional input parameter is the matrix elastic properties.Three sets of data were found in the literature upon which to validate this theory. These three sets all gave the matrix elastic moduli, the volume fraction and sieve analysis of the aggregate, and the elastic properties of the aggregates. All three data sets were for mortars. A data set for concrete that was complete enough to use to test the effective medium theory was not found. However, since the effect of the ITZ is less for concrete than for mortar, it is thought that mortar data supplies a more rigorous test for the theory than concrete data. There was good agreement between theory and experiment for all three mortars. The details of comparison, along with appropriate graphs, are given in Case Study II later in this report.

Additional work on the elastic properties of mortars was undertaken with Dyckerhoff, and with Prof. S.P. Shah at ACBM (Northwestern University). These data were not completely analyzed at the time of writing, and will be reported on next year. The equations incorporated into VCCTL 4.0 will allow the user to specify: matrix moduli, volume fraction of aggregate, elastic moduli of aggregate, size of the ITZ, elastic properties of the ITZ, and sieve analysis of the aggregates. A suggested use is to compute the cement paste elastic moduli using the desired cement, and then to input these values into the mortar/concrete option as matrix moduli so as to be able to compute the mortar/concrete moduli.

There are various empirical equations relating compressive strength and Young's modulus in the concrete literature (ACI, etc.). The purpose of these equations is 
really to derive the Young's modulus, useful in structural calculations, from the measured compressive strength. For VCCTL, since elastic moduli can be predicted, the use of equations like this is to predict the compressive strength, a useful number for quality control and performance, from the predicted elastic moduli. It turns out, via conversations with Prof. Franz-Josef Ulm (MIT), that the standard empirical equations, which relate the square root of strength to Young's modulus, do have some basis in strength of materials theory. A project has been initiated with Prof. Ulm to develop more precise versions of such equations that take into account the multi-scale nature of concrete, from the nano level (C-S-H) to the milli-level (concrete). This approach is based on nano-indentation of cement paste phases, and already has been successful in other, similar materials. An MIT M.S. student, funded by VCCTL, will spend 9 months at NIST starting in December, 2003, to implement this project. VCCTL 4.0 includes existing ACI empirical equations, empirically predicting compressive strength based on computations and predictions of elastic moduli. The next version, 5.0, hopefully will contain improved strength equations based on the work to be done in the $4^{\text {th }}$ year of the consortium.

\section{Future}

1. Establish and validate more accurate correlations between concrete elastic moduli and compressive strength.

2. Implement improved predictions of compressive strength within VCCTL software.

3. Improve early-age mechanical property predictions.

\section{References}

1. D.P. Bentz (editor) “The Virtual Cement and Concrete Testing Laboratory Consortium Annual Report 2001," NISTIR 6840, U.S. Department of Commerce, 2001.

2. E.J. Garboczi, "Finite Element and Finite Difference Programs for Computing the Linear Electric and Elastic Properties of Digital Images of Random Materials," NISTIR 6269, U.S. Department of Commerce, 1998. Available at http://ciks.cbt.nist.gov/monograph/manual/man.html .

3. A.P. Roberts and E.J. Garboczi, "Elastic Properties of Model Porous Ceramics," J. Amer. Ceram. Soc. 83, 3041-48 (2000). Available at http://ciks.cbt.nist.gov/monograph/Tonycer/Tonycer.html .

4. A.P. Roberts and E.J. Garboczi, "Elastic Properties of a Tungsten-Silver Composite by Reconstruction and Computation," J. Mech. and Phys. of Solids 47, 2029-55 (1999). Available at http://ciks.cbt.nist.gov/monograph/paper108/tungag.html .

5. A.P. Roberts and E.J. Garboczi, "Elastic properties of model random three-dimensional open-cell solids," J. Mech. Phys. Solids 50, 33-55 (2002). Available at http://ciks.cbt.nist.gov/monograph/opencell/oc02a.html . 
6. A.P. Roberts and E.J. Garboczi, "Computation of the linear elastic properties of random porous materials with a wide variety of microstructure," Proc. Roy. Soc. London A MAT 458, 1033-54 (2002). Available at http://ciks.cbt.nist.gov/monograph/paper127/b06.html .

7. E.J. Garboczi and J.G. Berryman, "Elastic moduli of a material containing composite inclusions: Effective medium theory and finite element computations," Mech. Mater. 33, 455-470, (2001). 


\section{Durability Research}

To design a concrete for a specified minimum life, it is necessary to understand the processes that cause deterioration, including the rates at which these will occur under the conditions to which it will be subjected [1].

\section{Background}

One of the ultimate goals of the VCCTL Consortium is the prediction of service life of concrete based on fundamental knowledge of the starting materials, mixture proportioning, curing conditions, and environmental factors. A moment's reflection will reveal how complex an exercise it is to make accurate predictions. The field durability of concrete depends on a myriad of phenomena that interact and compound, beginning with its initial placement. One must have a reasonably complete understanding of microstructure development during curing, and of the mechanisms by which the microstructure deteriorates due to environmental exposure.

\section{Activities in Year 2}

VCCTL research in durability has focused in Year 2 on the resistance of cement pastes to degradation by sulfate attack, and this work has proceeded along two complementary paths: development of a sulfate-resistance database and computational modeling of sulfate attack on cement paste.

\section{Research in Year 3}

The methodology adopted at NIST to determine the sulfate resistance of concrete under sulfate attack is based two major mechanisms: 1) penetration of sulfate ions into concrete; 2) chemical reaction of sulfate with the cement hydration products. The latter will trigger expansion and deterioration of the specimen. Under the sponsorship of FHWA and PCA, some parts of this approach are ready and can be made available to VCCTL. This approach is illustrated in Figure 8.

\section{Modeling}

- CONCLIFE is a model that assumes that once sulfates penetrate into the concrete, chemical reaction is initiated and deterioration occurs. This model is based on the measurement of the sorption coefficient (ASTM standard approved; to be issued in 2004). CONCLIFE-FHWA is available from the NIST website (http://ciks.cbt.nist.gov/ bentz/phpct/cmml.html)

- SULFATE2: a model predicting the expansion of concrete from its composition and from the geometry of the specimen was developed by Dr. 
Barzin Mobasher (Arizona State University). As per agreement with Mobasher, SULFATE2 is now available on the NIST website

- A semi-empirical model based upon cement phase composition and particle size distribution. Once validated, this model could predict the expansion of a mortar from mineralogical and the particle size distribution of the cement. This model is under development in collaboration with the NIST Statistical Engineering Division

- 4 SIGHT; a model that allows the calculation of the formation factor of a concrete, is also available from the NIST website (http://ciks.cbt.nist.gov/ bentz/phpct/cmml.html)

Progress was made in designing an accelerated test to determine the expansion of a mortar under sulfate attack. Specimen size was reduced from the standard ASTM C 1012 mortar bar, with a reduction in test time by a factor of four. This revised procedure is in the process of being correlated with C 1012 data.

Ultimately, with exposure conditions and mix design held constant, the performance of cement is based upon the phase composition and fineness.

Applying accurate means to measure these properties allows exploration of the sulfate performance relative to mineralogy and fineness, and identification of the significant variables in sulfate-resistant cements. Quantitative X-ray powder

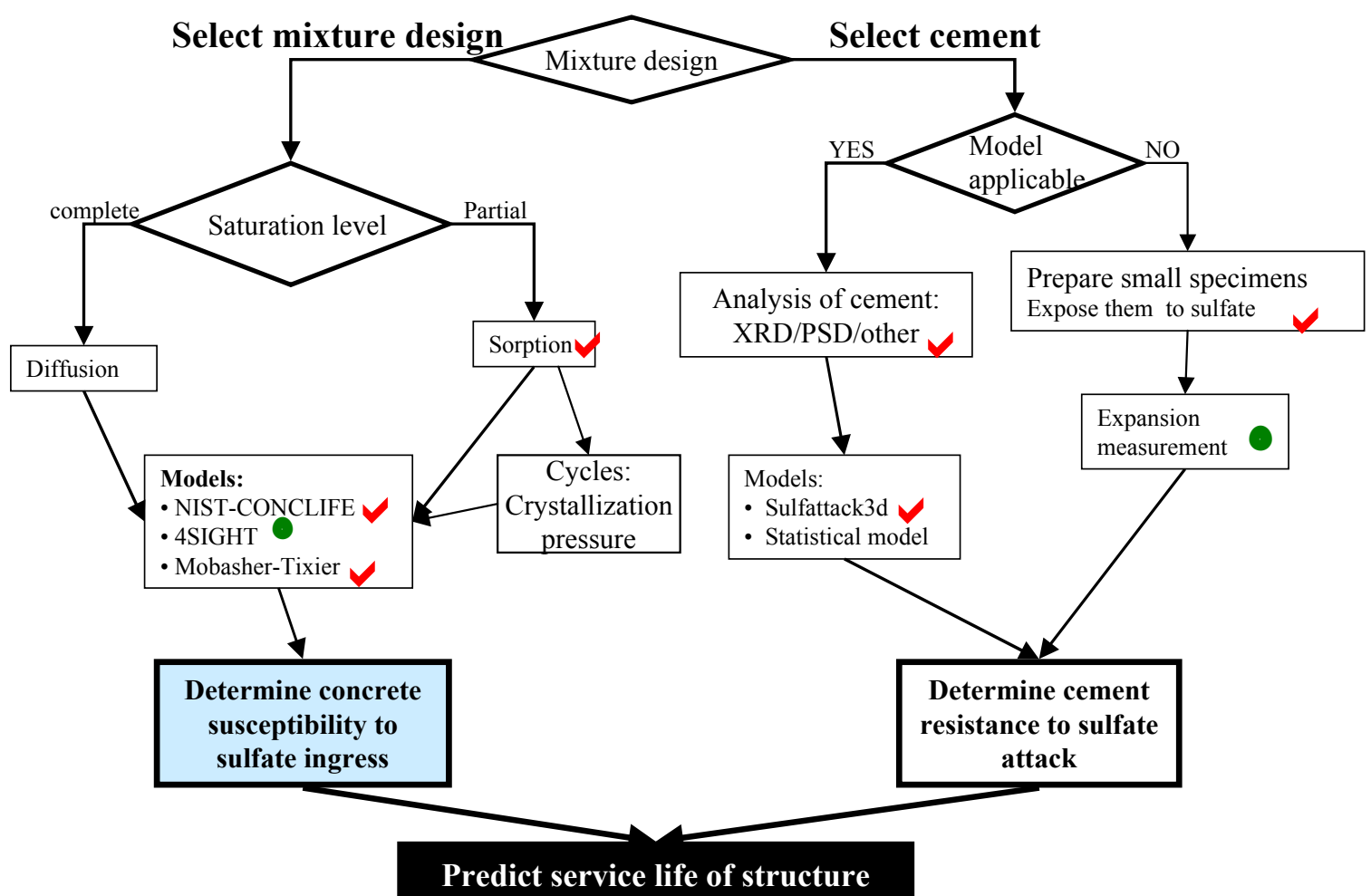

Figure 8: Schema of the sulfate resistance prediction. The check marks indicated areas that are completed or almost operational. The dots indicated areas where progress was made but further work is necessary 
diffraction analysis of cement mineralogy and SEM imaging of cement microstructure are being applied to explore performance - composition property relationships. Statistical analyses of the data are being used to identify the most significant combination of variables to predict C 1012 performance. Current results indicate both a phase composition and fineness component as significant and development of non-linear, multi-component models are expected to improve predictive capability.

\section{Future}

The development of an accelerated test for determining the resistance to sulfate attack of cement includes the use of smaller samples than the ones described in ASTM C 1202. This method will reduce the duration of the test by a factor of 3 to 5 . More validation tests should be conducted in VCCTL Phase II.

\section{References}

1. H.F.W. Taylor, p. 356 in Cement Chemistry. $2^{\text {nd }}$ Edition. Thomas Telford Publishing, London, 1997.

2. P. Stutzman, "Scanning Electron Microscopy Imaging of Hydraulic Cement Microstructure", to be published in Special issue of Cement and Concrete Composites on Microstructure, 2004

3. P. Stutzman, "Development of an ASTM Standard Test Method on X-Ray Powder Diffraction Analysis of Hydraulic Cements", Proceedings of the Denver of the X-Ray Conference, Denver 2003 


\section{Case Study I}

\section{Influence of Cement Particle Shape on Hydration Behavior}

New in VCCTL Version 4.0 is the ability to incorporate real-shape cement particles when creating a 3-D cement paste microstructure. Other than the qualitative differences in the appearance of the initial microstructure, it is an open question whether real particle shapes influence quantitatively the hydration kinetics of a cement paste.

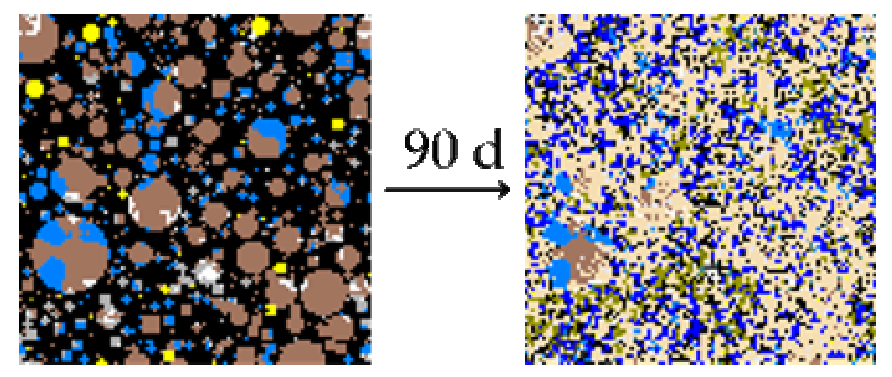

Figure 9: CCRL cement 116 microstructures created with spherical particles (top) and real-shape particles (bottom) at $w / c=0.4$ and hydrated for $90 \mathrm{~d}$ at $25^{\circ} \mathrm{C}$ under saturated conditions.
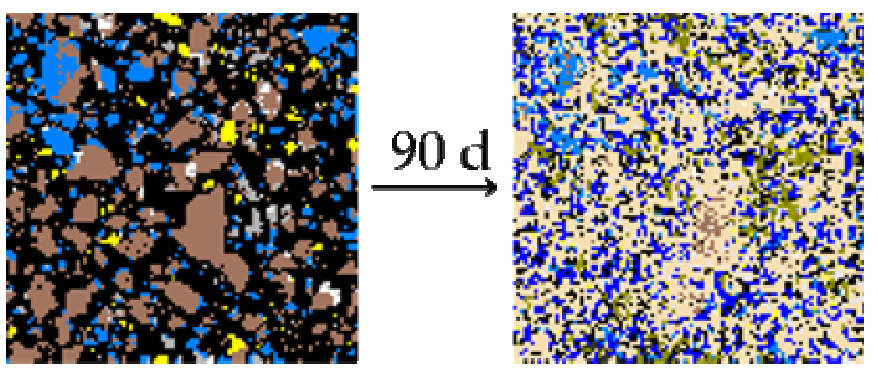

Figure 9 shows slices from beginning and $90 \mathrm{~d}$ microstructures predicted by CEMHYD3D for CCRL cement 116 with $\mathrm{w} / \mathrm{c}=0.4$ and cured isothermally at $25{ }^{\circ} \mathrm{C}$ under saturated conditions. The top row shows a microstructure

created with spherical particles and the bottom row shows a microstructure created with real-shape particles. The real shapes were obtained from CCRL cement 133 using the Visible Cement Data Set [1]. The same PSD was used to create both microstructures, and the cement phase proportions are the same to within \pm 0.00002 for the volume fraction of any given phase. Therefore, these two microstructures can be used to isolate the influence of particle shape on hydration behavior.

Although the two microstructures initially differ in appearance, there is very little qualitative difference in the $90 \mathrm{~d}$ microstructures in Fig. 9. Much of the particle shape information is lost as hydration proceeds. Based on this, one might expect that any differences in hydration behavior will be greatest at early ages.

Figure 10 shows both the degree of hydration and the fraction of connected solidsan indication of setting time-as a function of age. The circles are experimental measurements of the degree of hydration based on nonevaporable water content. . 
Both curves in the first plot intentionally have been fit, by adjusting their $\beta$ parameters, to match the experimentally measured degree of hydration at $8 \mathrm{~h}$. Negligible differences in the degree of hydration are observed at all ages.

One significant early-age property that does seem to be slightly affected by particle shape is the onset of setting, as shown in Fig. 10. The system with spherical particles forms a tenuously connected solid network at $6 \mathrm{~h}$, while the system with real-shape particles begins to percolate at $3 \mathrm{~h}$. As the first plot in Fig. 10 shows, this difference is not due solely to differences in hydration kinetics, but instead must be attributed, at least partially, to a particle shape effect. Spheres are isometric and have an aspect ratio of unity, and any departure from sphericity using particles with higer aspect ratio, is well known to decrease the percolation threshold of hydration products. Nevertheless, the effect produced is quite minor, and the curves for both microstructures converge such that by eight hours they are virtually identical.
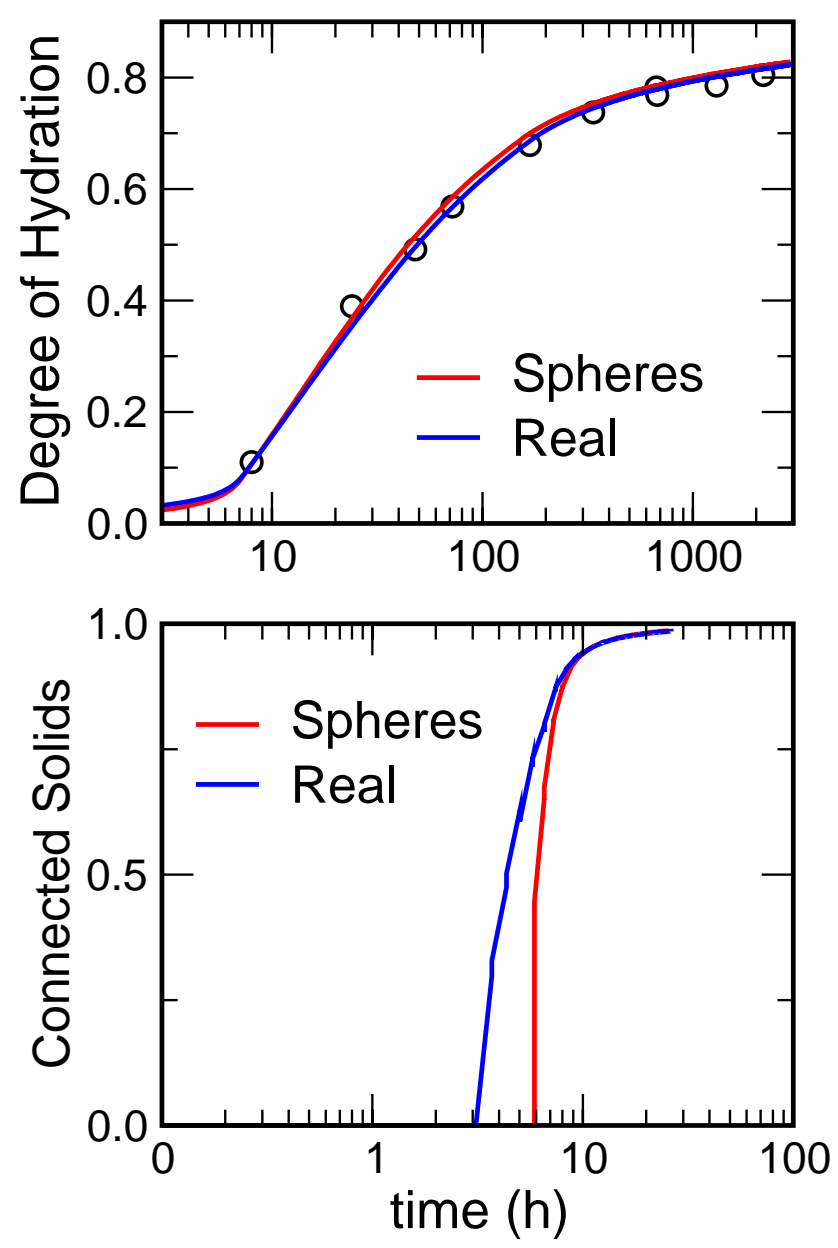

Figure 10: Degree of hydration and fraction of connected solids as a function of age for CCRL cement 116 microstructures shown in previous figure 


\section{Case Study II}

\section{Validation of effective medium theory for prediction of mortar and concrete elastic moduli based on knowledge of individual component moduli}

The following three sets of data were used to validate the effective medium theory calculations of mortar elastic moduli:

[1] A. Boumiz, "Etude comparèe des evolutions mechaniques et chimiques des pates de ciment et mortiers à très jeune age", French Ph.D. thesis, 1995.

[2] Zimmerman, King, and Monteiro, "The elastic moduli of mortar as a porousgranular material," Cem. Conc. Res. 16, 239-245 (1986).

[3] O. Ishai, "Influence on sand concentration on deformations of mortar beams under low stresses," J. of ACI 58, 611-622 (1961).

\section{Boumiz data}

This mortar had small sand (quartz) particles, only $8 \mu \mathrm{m}$ to $300 \mu \mathrm{m}$ in diameter. For this size sand particles, the ITZ is not well-defined for most particles, since the aggregate size is on the same order as the cement size. Therefore, no ITZ was used in the effective medium theory (EMT). The air content was reported for each amount of sand, and was incorporated in the EMT. The effect of air can be substantial - it can lower the elastic moduli by a percentage amount equal to roughly three times the volume percentage of air. The mortar had w/c $=0.5$, and was hydrated for $28 \mathrm{~d}$.

\section{Zimmerman and Monteiro data}

The amount of air was not measured, hence was not included in the calculation. The mortar was said to be "well-hydrated," with $w / c=0.35$. The aggregate was quartz aggregate, with $\mathrm{K}=37 \mathrm{GPa}$, and $\mathrm{G}=44 \mathrm{GPa}$. The effective ITZ was assumed to be $0.01 \mathrm{~mm}$ in thickness. The Young's modulus of the ITZ was taken to be half that of the bulk matrix paste $\left(\mathrm{E}_{\text {ITZ }}=0.5 \mathrm{E}_{\text {paste }}\right.$, and the Poisson's ratio of bulk and ITZ paste taken to be the same. The measured wave speeds were read off a graph, and converted to elastic moduli (GPa) using the measured densities. 


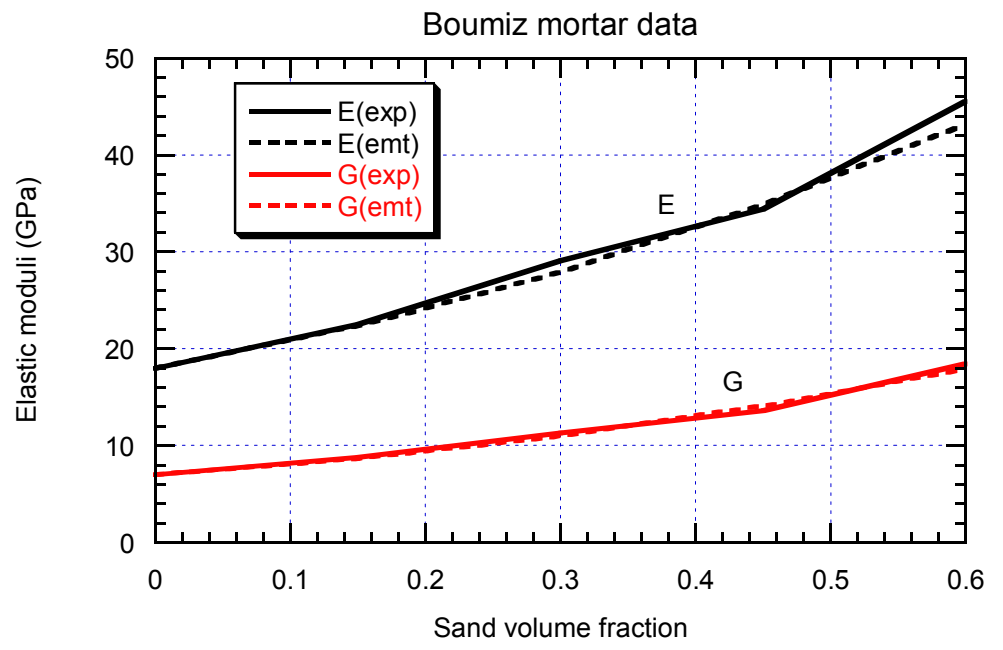

Figure 11: Boumiz mortar data

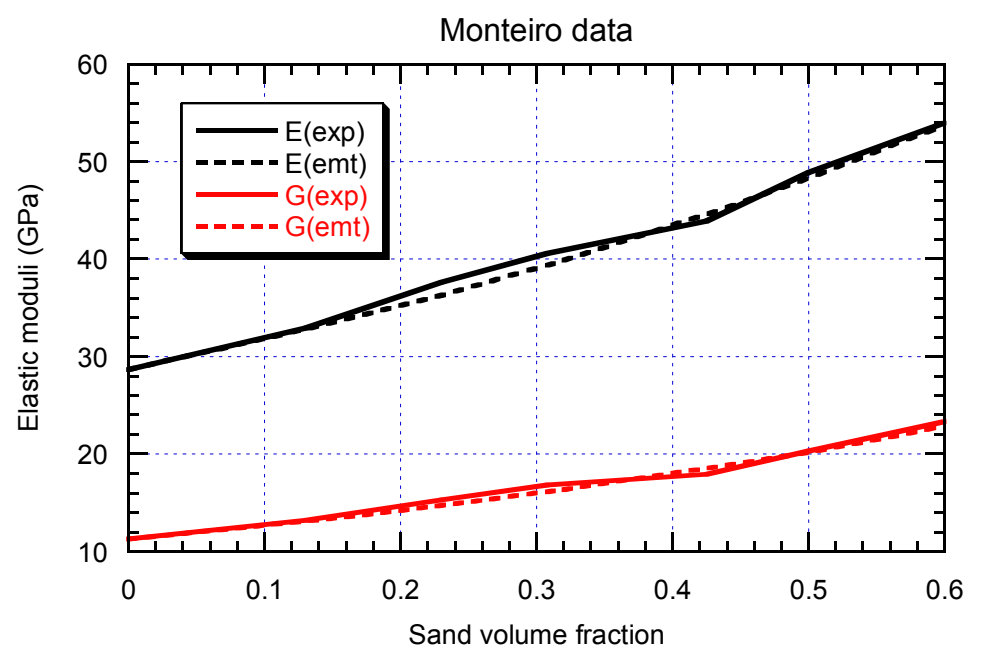

Figure 12: Monteiro data

\section{Ishai data}

Data were listed in terms of fitted straight lines, so the experimental data appears as straight lines in the graph below. No amount of air was reported, so no amount of air was allowed in the EMT calculation. The value of $w / c=0.32$ was used, with $28 \mathrm{~d}$ sealed curing reported. A "Standard Leighton Buzzard sand" was used. This is quartz sand. The sieve analysis was assumed to be the same as ASTM graded standard sand (between $0.15 \mathrm{~mm}$ and $0.6 \mathrm{~mm}$ ). The effective ITZ thickness was taken to be $0.01 \mathrm{~mm}$, with $\mathrm{E}_{\mathrm{ITZ}}=0.5$ Epaste, and the Poisson's ratio of bulk and ITZ paste taken to be the same.

In all three cases, there was good agreement between theory and experiment. This EMT will become part of VCCTL 4.0. 
Ishai data

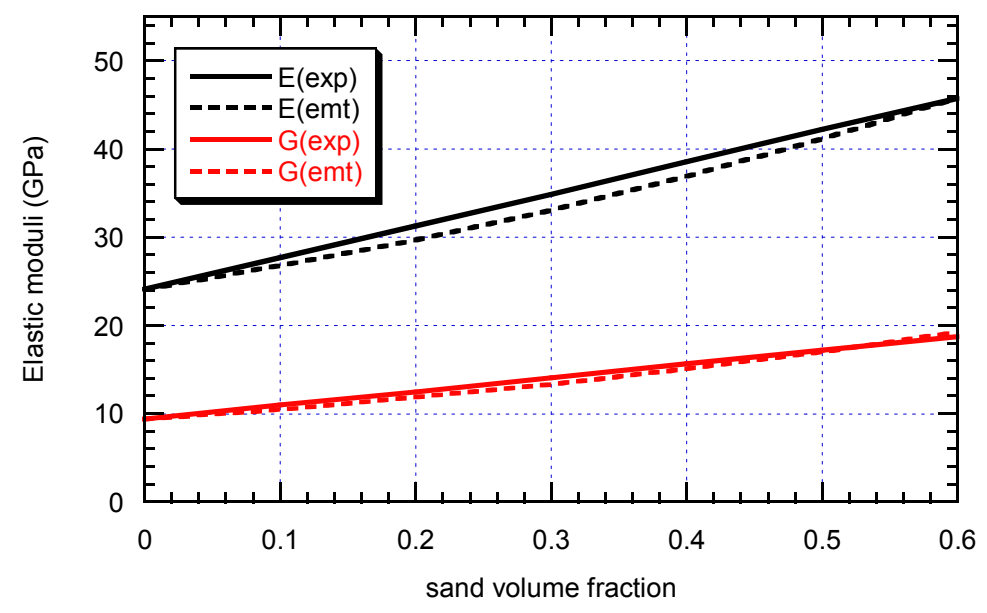

Figure 13: Ishai data. 


\section{Case Study III}

\section{Effect of Aggregate Size Distribution on Concrete Plastic Viscosity}

The role of size distribution of spherical shaped aggregates on relative viscosity was investigated in collaboration with Dr. Robert Flatt (Sika). This work will form the basis for an optimization of aggregate gradation for flow performance.

In this study, the particle size distributions were characterized by $\sigma$ as defined by the following equation:

$$
\sigma=\frac{1}{<r>} \sqrt{\frac{\sum\left(r_{i}-<r>\right)^{2}}{N}}
$$

where $\langle r\rangle=\frac{\sum r_{i}}{N} ; r_{\mathrm{i}}=$ particle radius; $N=$ number of particles, so that $\langle r\rangle$ is the number-weighted average particle radius. The parameter $\sigma$ is a measure of the width of the size distribution.

In this definition, the particle size distribution is normalized by its average size. By this approach, the results can be applied to a broader range of aggregates. For this study, we considered $\sigma$ varying from 0 to 1 . A value of $\sigma$ equal to zero indicates that the spheres are mono-size. A value of $\sigma$ equal to 1 means that the sphere radii varied by a factor of 30 . Figure 14 shows pictures of a realization of two different particle size distributions (PSD), characterized by two different values of $\sigma$.

As a case study, we selected several lognormal particle distributions. Figure 15 shows the distribution selected. In Figure 15, a standard silica sand (ASTM C778) was also included. Note that, when the sand data is properly normalized, the sand has a value of $\sigma=0.6$. These simulations involved the inclusion of thousands of particles, which up to now would have been nearly impossible by conventional methods. Indeed, to the best of our knowledge there are no previous simulations of poly-disperse suspensions. Therefore, this work represents a real advance in the study of the rheology of suspensions.

To determine the influence of aggregate size distribution on the relative viscosity, the suspensions were sheared using a constant strain rate. The stresses in the system were then calculated and used to determine the viscosity of the suspension. Figure 16 shows the relative viscosity as a function of solid fraction for the different values of $\sigma$ shown in Figure 15. Note that at low solid fractions there is not much difference in the data for different $\sigma$. However, as the solid fraction increases, the 
relative viscosity clearly decreases with increasing $\sigma$. This can largely be understood as resulting from the maximum packing of the sphere system increasing as the PSD become wider. The solid lines in Figure 16 are fits to the data, based on the following semi-empirical equation constructed by Jack Douglas,

$$
\frac{\eta_{\mathrm{c}}}{\eta_{0}}=\left(1-\frac{\mathrm{C}}{\mathrm{C}^{*}}\right)^{-2} \cdot\left[1-0.4\left(\frac{\mathrm{C}}{\mathrm{C}^{*}}\right)+0.34\left(\frac{\mathrm{C}}{\mathrm{C}^{*}}\right)^{2}\right]
$$

where $C$ is the aggregate concentration, $C^{*}$ is the maximum aggregate packing, and $\eta_{c} / \eta_{0}$ is the relative viscosity. The only free parameter in this equation is the maximum packing fraction, $C^{*}$. This equation provided fits somewhat better than the commonly used Krieger-Dougherty equation.

These results have been included in the newest version of the VCCTL software. In the future, we plan to extend this study to included non-spherical particles based on the VCCTL database of real shaped aggregates that were derived from X-ray tomography measurements.

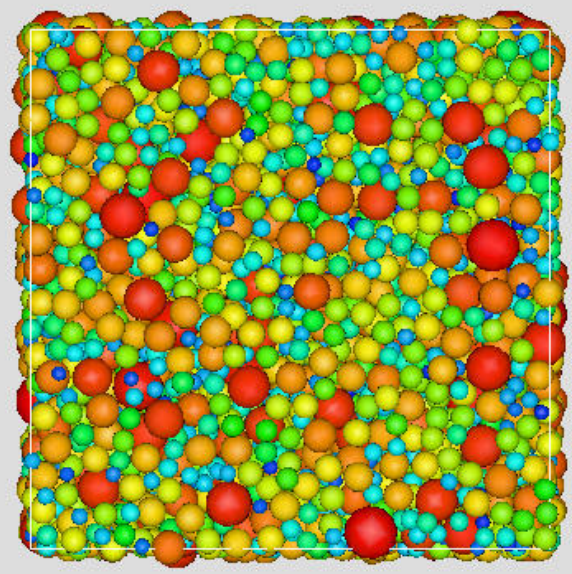

$\sigma=0.2$

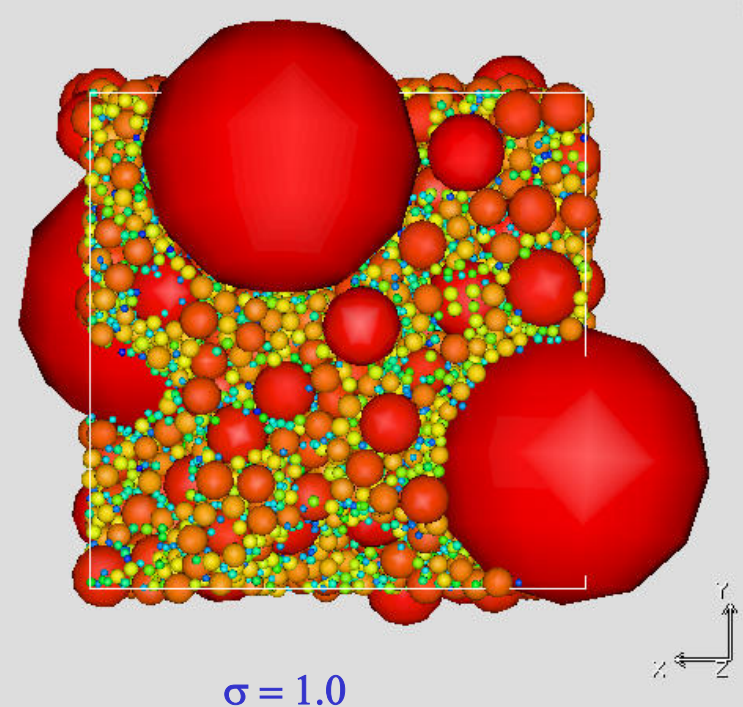

Figure 14: Various particle size distributions. See text for a definition of $\sigma$. 


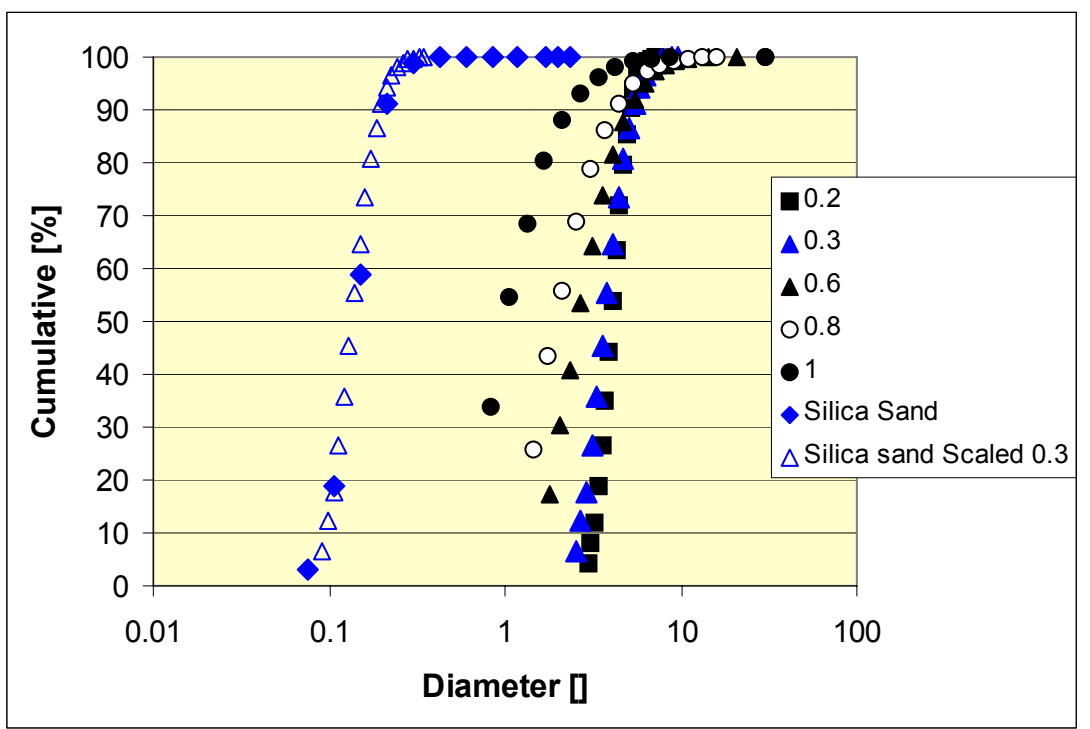

Figure 15: Distribution of aggregates with varying $\sigma$ used in this case study.

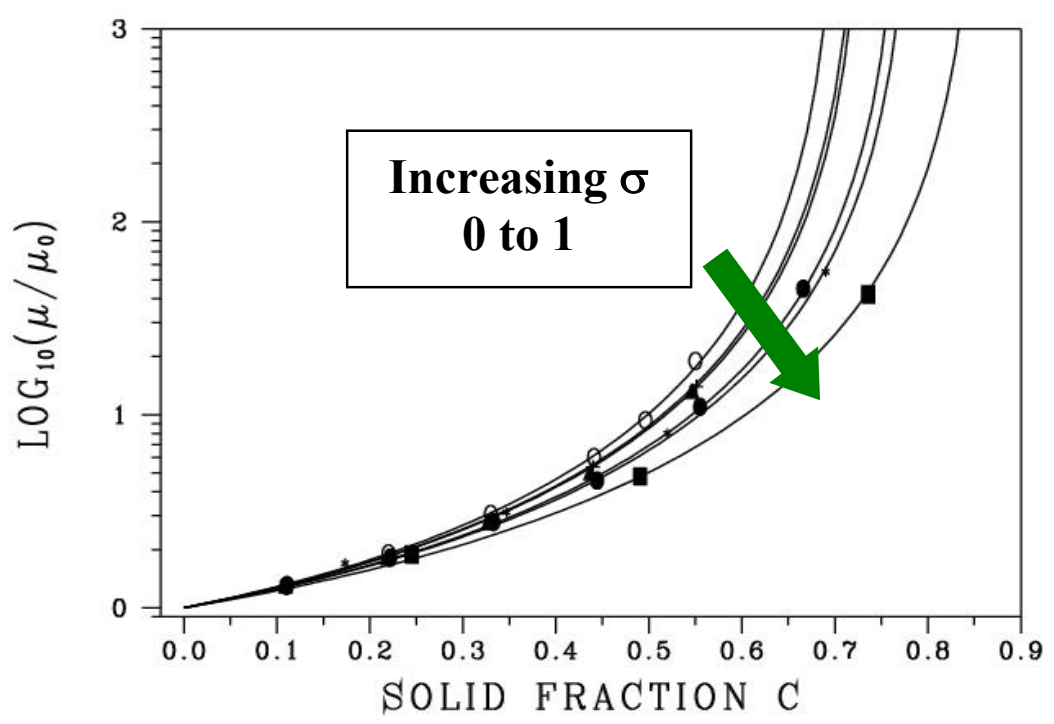

Figure 16: Aggregate concentration and distribution influence on relative viscosity 


\section{NIST/Industry VCCTL Funding}

External funding of VCCTL (from consortium members outside NIST)

$\$ 0.40 \mathrm{M}(7 \quad \$ 40 \mathrm{~K}+2 \quad \$ 60 \mathrm{~K})$

Internal NIST funding of VCCTL research activities

$\$ 0.85 \mathrm{M}(\mathrm{BFRL})+\$ 0.55 \mathrm{M}(\mathrm{ITL})=\$ 1.4 \mathrm{M}$

NIST equipment funding

BFRL - New scanning electron microscope - \$250K

BFRL - X-ray tomography at Penn State - \$25K

ITL - New floor to RAVE, three new projectors - \$215K

Total $=\$ 0.49 \mathrm{M}$

Total NIST funding of VCCTL project (labor + equipment)

$\$ 1.89 \mathrm{M}$

Total VCCTL project funding

$\$ 2.29 \mathrm{M}$

Percentage shares of total VCCTL project funding

NIST $-82 \%$

Industry $-18 \%$

Total cost/industrial share $\quad 5.6: 1$ 


\section{NIST Equipment and Facilities}

\section{Materials and Construction Research Division}

\section{Microscopy}

- Scanning electron microscope equipped with solid-state backscattered electron detector. Digital image capture, processing and analysis capabilities. X-ray microanalysis capabilities for qualitative and quantitative spot analyses as well as imaging element spatial distribution. (New machine in 2003)

- Automated X-ray powder diffractometer with nine-position sample changer, adjustable optics, diffracted beam monochromator

- Light Microscopy Facilities:

- Stereo microscope utilizing apochromatic objectives and single optic axis

- Polarized light petrographic microscope

- Reflected/transmitted light microscope

- Atomic force microscope utilizing an environmental chamber

- Confocal microscope

- Specimen preparation facilities for SEM, optical microscopy, XRD and XRF.

- Image Processing: including digital and optical camera. Semi-automated image analysis is available

\section{Mechanical Properties}

- Compressive testing machines

- Nano-indenter

- Stress measurements of a confined specimen for ASR or sulfate attack studies

\section{Transport Properties}

- Gas permeability equipment 
- X-ray absorption unit for monitoring water movement in materials (to be upgraded in 2004)

- Electrical conductivity measurement equipment

- Ionic diffusivity measurement equipment

\section{Systems Characterization}

- Malvern particle size analyzer, wet and dry capability

- Particle classifier (new in 2003)

- Quantachrome AUTOSORB-1, a multi-point surface analysis system for BET surface area and porosity (acquired in 2003)

- Quantachrome AUTOSCAN 60 for mercury porosimetry (acquired in 2003)

- Atomic absorption

- Infrared spectrometer

- Ion chromatograph

- Thermogravimetric analysis

- Differential scanning calorimeter (DSC)

- Differential thermal analysis (DTA)

- Automated high-temperature furnace to $1600{ }^{\circ} \mathrm{C}$

- Dunouy Tensiometer for measurement of the surface tension of solutions.

- Heiden Sorption Analyzer for measurement of absorption/desorption isotherms.

\section{Rheology}

- Parallel plate fluid rheometer: stress or strain controlled

- Controlled temperature and speed mixer: This mixer is designed according to the specifications developed by PCA/CTL and can be used for paste and/or mortar

- Standard tools for flow measurement in cement paste or mortar, like Marsh cone, flow cone, mini slump

\section{Sample Preparation Laboratory}

- Attritor mill 
- Fully equipped laboratory to prepare and cure mortar or cement paste specimens including Hobart mixers, flow table, vibrating table

- Environmental chambers:

- three cabinets (temperature and RH controlled)

- one walk-in (temperature controlled)

- curing cabinet (temperature controlled and RH higher than $98 \%$ )

- Water baths: 4 table top and one floor unit

- $\mathrm{pH}$ controlled units

\section{Computational Facilities (Materials and Construction Research Division)}

- Windows and LINUX-based PCs

- 32-processor Linux cluster

- 128-processor Linux cluster (new in 2003)

- 64-processor Linux cluster (new in 2003)

\section{Information Technology Laboratory Central Hardware Facilities (ITL)}

\section{Computational Resources}

- Four SGI Origin 2000 machines:

- 8196 Mhz R10000 CPUs, 8 GB of memory, 120 GB of disk space

- 32250 Mhz R10000 CPUs, 32 GB of memory, 96 GB of disk space

- 32300 Mhz R12000 CPUs, 32 GB of memory, 193 GB of disk space

- 32300 Mhz R12000 CPUs, 32 GB of memory, 193 GB of disk space

- One SGI Cluster

- five dual processor R10000 CPUs, with 4 GB of memory

- Three Linux Clusters

- sixteen $400 \mathrm{MHz}$ Pentium IIs connected by a Fast Ethernet network.

- $\quad$ Each CPU has $256 \mathrm{MB}$ of RAM and 6GB of local disk storage.

- forty-eight 500Mhz Pentium IIIs connected by a Fast Ethernet network. 
- Each CPU has at least 256MB of RAM and 6GB of local disk storage.

- 128 Pentium IIIs connect by a Fast Ethernet network

- Each CPU has 1GB of RAM and 6GB of local disk storage

\section{Visualization Resources}

- $\quad$ One SGI Onyx3000

- Four Infinite Reality4 Graphics

- 24 R14000 CPUs (500 MHz)

- 24 GB memory

- One RAVE two-wall immersive environment with Crystal Eyes software/hardware with head tracking 


\section{For More Information}

- Examine Version 1.1 of the VCCTL software package, available at:

http://vcctl.cbt.nist.gov .

- Visit the VCCTL Consortium information web site at:

http://www.bfrl.nist.gov/862/vcctl

- Contact the VCCTL Consortium Manager at:

Dr. Jeffrey W. Bullard

National Institute of Standards and Technology

100 Bureau Drive Stop 8615

Gaithersburg, MD 20899-8615 USA

Phone: 301.975 .5725

Fax: $\quad 301.990 .6891$

bullard@nist.gov 